\title{
Characteristics of Thermal and Suprathermal Ions Associated With the Dayside Plasma Trough as Measured by the Dynamics Explorer Retarding Ion Mass Spectrometer
}

\author{
J. J. SOJKA AND R. W. SCHUNK
}

Center for Atmospheric and Space Sciences, Utah State University

\author{
J. F. E. Johnson, J. H. WAITE, AND C. R. ChAPPELL \\ Space Science Laboratory, Marshall Space Flight Center
}

\begin{abstract}
The retarding ion mass spectrometer (RIMS) instrument on Dynamics Explorer 1 has carried out high-altitude measurements of the polar wind. During an outbound pass through the outer plasmasphere and plasma trough at 0900 LT the RIMS instrument measured the detailed characteristics of the thermal and suprathermal ions. Three distinct populations were observed: (1) thermal ions $\left(\mathrm{H}^{+}\right.$and $\left.\mathrm{He}^{+}\right)$with densities ranging from $>500$ ions $/ \mathrm{cm}^{3}$ to $<1 \mathrm{ion} / \mathrm{cm}^{3}$, temperatures around $2 \mathrm{eV}\left(20,000^{\circ} \mathrm{K}\right)$, and $\mathrm{H}^{+} / \mathrm{He}^{+}$density ratios of 2 to 4 , (2) warm anisotropic plasma with densities ranging from 2 to 12 ions $/ \mathrm{cm}^{3}$, temperatures between 10 and $15 \mathrm{eV}$, and $\mathrm{H}^{+} / \mathrm{He}^{+}$density ratios of 0.1 to 6 , and (3) thermal field-aligned $\mathrm{H}^{+}$and $\mathrm{He}^{+}$ion streams displaying both single stream and counterstreaming flow characteristics-the polar wind. These streaming ions display temperatures $\leqslant 1.5 \mathrm{eV}$ and $\leqslant 2.0 \mathrm{eV}$ for $\mathrm{H}^{+}$and $\mathrm{He}^{+}$, respectively, and respective parallel drift velocities of $<30 \mathrm{~km} / \mathrm{s}$ and $<15 \mathrm{~km} / \mathrm{s}$ with an $\mathrm{H}^{+} / \mathrm{He}^{+}$flux ratio of 3 to 10 . Best-fitting techniques for the $\mathrm{H}^{+}$and $\mathrm{He}^{+}$streaming distributions indicate a supersonic flow, although the data cannot be interpreted unambiguously. These comprehensive measurements of thermal and suprathermal plasma characteristics are interpreted in terms of plasmasphere refilling processes.
\end{abstract}

\section{INTRODUCTION}

After a magnetic storm the outer plasmasphere is left in a state of lower density relative to its prestorm state. Over a period of one to several days, depending upon the severity of the storm, this region is refilled. The general characteristics of the refilling rate have been inferred from whistler observations [Carpenter and Park, 1973; Park, 1974] and OGO 5 plasma measurements [Chappell, 1972]. This refilling process is assumed to be caused by plasma from the ionosphere expanding along magnetic flux tubes into the depleted plasmasphere, although no in situ measurements have been made of the characteristics of the upstreaming thermal ions that are responsible for the refilling process. That such processes do occur has been inferred from time dependent increases in the isotropic thermal plasma by the ISEE 1 plasma detector [Horwitz et al., 1981b]. Since the geomagnetic field lines are primarily dipolar, the plasma flows from both the northern and southern ionospheres toward the equator where they meet. At higher latitudes this outflow of plasma from the ionosphere into a depleted magnetosphere is referred to as the polar wind.

Theoretical models of both a kinetic and hydrodynamic nature have been used to study the expansion of ionospheric plasma to higher altitudes along magnetic flux tubes [Banks and Holzer, 1969; Marubashi; 1970 Holzer et al., 1971; Lemaire and Scherer, 1974; Raitt et al., 1977; Schunk and Watkins, 1982]. These models in general allow for both subsonic and supersonic outflow

Copyright 1983 by the American Geophysical Union.

Paper number $3 \mathrm{~A} 1148$.

0148-0227/83/003A-1148\$05.00 velocities for the ionospheric plasma as it enters the collisionless regions above 2000 to $3000 \mathrm{~km}$. The particular character of the outflow is very sensitive to the boundary conditions applied to the model [Schunk and Watkins, 1982]. Unfortunately, very limited data are available concerning these boundary conditions. In the lower collisional regions, the light ion outflow fluxes have been observed [Hoffman and Dodson, 1980]; however, these observations are not able to uniquely define the outflow characteristics. At higher altitudes, where the plasma density is lower, a variety of spacecraft effects, mainly that of vehicle potential, have made measurements of the thermal plasma component exceedingly difficult. In the outer plasmasphere, observations of anisotropic warm (a few electron volts to tens of electron volts) ions have been made [Bezrukikh and Gringauz, 1976; Horwitz and Chappell, 1979; Horwitz et al., 1981a, 1982; Chappell, 1982; Chappell et al., 1982; Gurgiolo and Burch, 1982]. Such warm plasmas and their associated pitch angle distributions indicate the presence of significant plasma heating perpendicular to the magnetic field, and these observations cannot be explained with the existing ionospheric outflow models. These anisotropic warm ions are evidence for wave particle interactions in the collisionless regions of the flux tube, and particularly in the equatorial region where the counterstreaming ionospheric plasmas meet.

It is the purpose of this paper to examine the thermal and suprathermal ion populations present in the refilling regions after a magnetic storm. Data from the Dynamics Explorer 1 spacecraft (DE-1) will be shown to display a variety of ion distributions in this region of space. These data were obtained by the retarding ion mass spectrometer (RIMS) experiment on DE-1 on October 14, 1982 as the satellite left the dayside plasmasphere and cut 
through the dayside mid-high latitude magnetosphere. This single data set is particularly useful in that an atypical instrument bias voltage enabled the screening effects of the spacecraft potential to be overcome.

\section{INSTRUMENT}

The RIMS instrument consists of three separate sensors, one mounted to view radially, and one mounted to view parallel $(+Z)$, and antiparallel $(-Z)$ to the spacecraft spin axis. Each sensor head consists of a retarding potential analyzer (RPA) followed by a magnetic mass analyzer with two separate exit slits corresponding to two mass ranges in the ratio 1:4. The three sensor heads are identical except that the $\pm Z$ sensors have a $\pm 55^{\circ}$ conical field of view, while the radial sensor has a rectangular angular acceptance of $\pm 10^{\circ}$ and $\pm 55^{\circ}$ in the planes perpendicular to and containing the spin axis, respectively. Ambient ions enter the sensor heads through the front apertures into retarding potential analyzer sections. The front aperture potential may be commanded to any of four values $(0,-2,-4$, or $-8 \mathrm{~V})$ to bias against a nonzero spacecraft potential. Indeed, it is this capability that allowed the positive spacecraft potential to be overcome and enabled the thermal ion population to be observed. The RPA retarding grid voltage is programmable over a 0 to $51 \mathrm{~V}$ range. The ions passing into the mass analyzer are accelerated and then sorted according to their atomic mass per unit charge. Ions of mass 1 to $8 \mathrm{amu}$ and 4 to $32 \mathrm{amu}$ can be focused on the low and high mass slits, respectively. Ions exiting the collector slits are counted by the channel electron multiplier detectors. For the data sets used in this paper the ion mass settings were selected for $\mathrm{H}^{+}$and $\mathrm{He}^{+}$ions on the low and high mass slits, respectively. The basic instrument cycle is 32 mass or energy steps over a period of $0.5 \mathrm{~s}$ with a basic data accumulation period of 12 ms. Further details and a more complete description of the RIMS instruments on DE-1 are given by Chappell et al. [1981].

\section{ObSeRVATIONS}

Figure 1 shows the DE-1 orbital and magnetic field geometry on October 14 and 15, 1981, starting at 2256 UT on the 14th through to 0045 UT on the 15th. The upper panel shows the projection on the noon-midnight geographic plane with the $Z$ GEI coordinate along the north geographic pole. On October 14 the sun had a declination of $-7.7^{\circ}$ and lay in the $X-Z$ plane, as indicated in the upper panel. The lower panel shows the projection onto the geographic $X \cdot Y$ (equatorial) plane. Over the period of interest, DE-1 moved to higher latitudes almost perpendicularly to the magnetic field lines from an $L$ value of 3 to one that lies in the vicinity of the dayside cusp region $(L>10)$. Three groups of field lines have been drawn from the spacecraft to the northern ionosphere, each group corresponding to a period of continuous RIMS data. These field lines are drawn at the time when the RIMS aperture bias was set to $-8 \mathrm{~V}$. At these times it was possible to overcome the positive spacecraft potential and observe thermal ions. Hence this set of locations, labelled $T_{1}$ to $T_{17}$, represents the times at which thermal

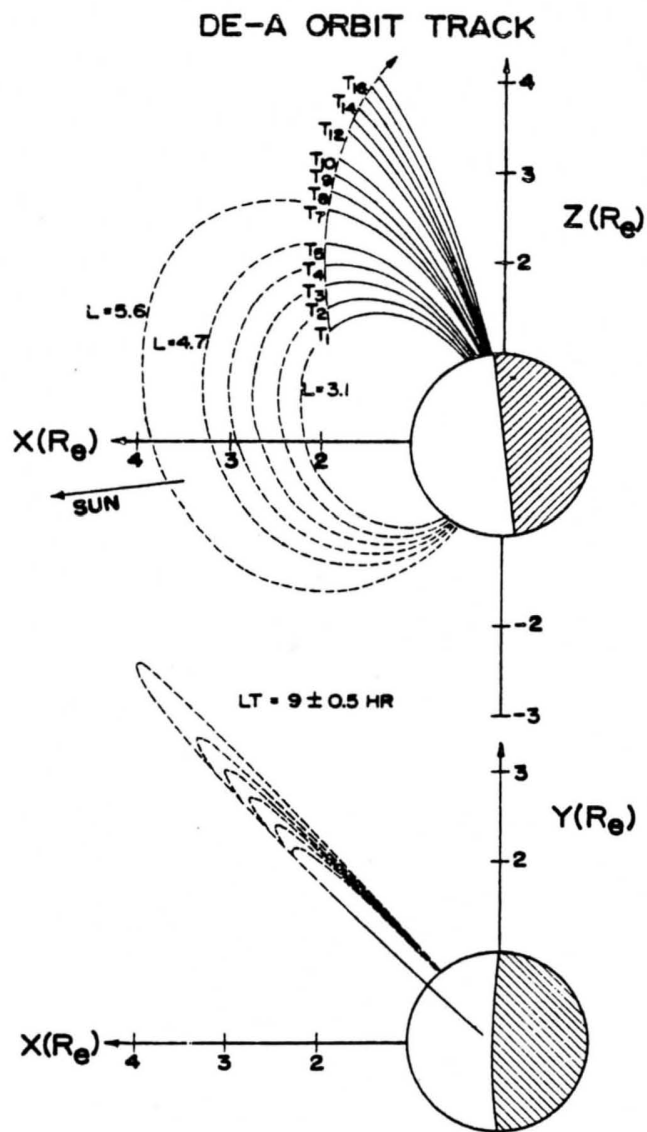

Fig. 1. Projections of the DE-A orbit and magnetic field in an inertial geographic coordinate system for the period 2256 UT on October 14, 1981, to 0045 UT on October 15, 1981. The magnetic field lines are shown at the times the RIMS instrument is in its $-8 \mathrm{~V}$ bias mode. Solid lines show the field lines mapping into the northern hemisphere, while dashed lines show the mapping to the conjugate hemisphere. The solar declination of $-7.7^{\circ}$ is indicated in the upper panel, where the projection is into the geographic noon plane. In the lower panel, the projection is into the equatorial X-Y plane.

ion characteristics can be studied. Table 1 lists the spacecraft location and velocity as well as the magnetic field line characteristics, which includes the spacecraft source cone angle for plasma coming adiabetically from an altitude of $2000 \mathrm{~km}$ in the northern hemisphere. The dashed field lines in Figure 1 trace the northern hemisphere field lines to their conjugate point in the southern hemisphere. The field line-tracing is based upon a tiltdependent magnetic field model (W. P. Olson and K. A. Pfitzer, unpublished manuscript, 1977). From Figure 1 it is evident that the orbit is ideal for observing the dayside field lines along which refilling occurs, since all these field lines are crossed at a fixed magnetic local time relative to plasma outflowing from the northern ionosphere. The upper panel in Figure 1 shows that the northern hemisphere field line enters the ionosphere near the terminator, but still in sunlight; hence, solar EUV ionization is still occurring on these field lines in the ionosphere. In addition, a major magnetic storm had reached its peak at about the time these measurements were made, which indicates that the outer plasmasphere was probably depleted. 


\section{$\infty \infty 0 \infty \infty$ \\ 응의

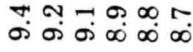

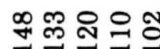

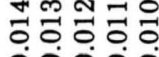

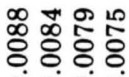

00000

$00 \div 0$

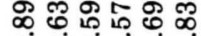
$\infty$ oi்

เ เค่ โก คิ

نூ

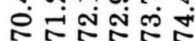

\section{$5=\infty$ \%}

\section{ํำ 유 लं लि लं}

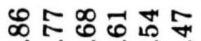

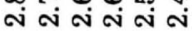

\section{망요 윰

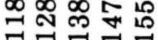

\section{드류요}

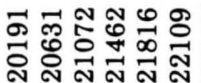

Figure 2 shows the $0.5 \mathrm{~s}$ average ion count rate of the RIMS radial analyzer at two energies for $\mathrm{H}^{+}$and $\mathrm{He}^{+}$during the pass. The upper panel shows the count rate at a retarding potential of $0.1 \mathrm{~V}$, while the lower panel is for a potential of $50 \mathrm{~V}$. These data are plotted linearly against time. Also shown above the upper panel are the times $T_{1}$ to $T_{17}$, at which the instrument was in the $-8 \mathrm{~V}$ aperture mode. Using these markers as a reference, it is possible to see the effect of changing aperture bias voltage upon the observed count rate. At the low retarding potential, 0.1 $\mathrm{V}$, the count rate displays a marked steplike variation on a time interval of 7.8 records; however, at the $50 \mathrm{~V}$ potential this steplike modulation is not present. The steplike variations follow the aperture bias cyclic sequence of $0 \mathrm{~V}$, $-2 \mathrm{~V}, 0 \mathrm{~V},-4 \mathrm{~V}, 0 \mathrm{~V},-8 \mathrm{~V}$, where each voltage is maintained for approximately $1 \mathrm{~min}$. As the bias becomes more negative, more thermal ions are able to penetrate the positive sheath around the spacecraft and enter the RIMS instrument, which increases the count rate. At the start of the data set the $0.1 \mathrm{~V}$ count rate is high, corresponding to a high thermal density and a spacecraft potential of about $0 \mathrm{~V}$ with respect to the plasma potential. However, as the spacecraft moves from the plasmasphere to the outer magnetosphere, the thermal density decreases and the spacecraft potential increases to values as high as $6.5 \mathrm{~V}$. with the increasing spacecraft potential there is an increase in the modulation depth associated with the aperture bias cycle; the ratio of count $(-8 \mathrm{~V}$ bias $)$ /count $(0 \mathrm{~V}$ bias) is $\sim 2$ at 2259:05 UT and increases to about 6 at 2345:45 UT. This changing modulation is consistent with the increased spacecraft potential being able to screen the spacecraft from a significantly larger part of the thermal plasma component, which acts to reduce the count rate at small and zero aperture biases.

At the $50 \mathrm{~V}$ retarding potential, lower panel of Figure 2, the count rate characteristics are somewhat different. The $\mathrm{He}^{+}$average count rate remains at the noise level, while the $\mathrm{H}^{+}$count rate increases markedly at 2257:05 UT and again at 2307:45 UT before declining until 2429:45 UT, where a final increase is observed. This final increase for fluxes at energies above $50 \mathrm{~V}$ is associated with a decline in the bias voltage modulation of the $0.1 \mathrm{~V}$ data and corresponds to a region where a warm isotropic ion population dominates other thermal ion populations. From a magnetospheric viewpoint, the data set covers four regions: (1) the outer plasmasphere (2256:33 UT to 2257:25 UT), which is dominated by a thermal $(\leqslant 2 \mathrm{eV})$ ion plasma, (2) the plasmapause (2257:53 UT to 2323:05 UT), where a mixture of thermal $(\leqslant 2 \mathrm{eV})$ and warm $(\leqslant 20 \mathrm{eV})$ plasma is present, (3) the low-density dayside magnetosphere (2323:05 UT to 2429:45 UT) of uncertain density and temperature, and (4) the CUSP region (2429:45 UT onwards), where only warm plasma ( $\geqslant 20 \mathrm{eV}$ ) is present. In the following subsections the detailed plasma composition, temperature, and isotropy will be described in each of these four regions.

\subsection{Outer Plasmasphere}

Figure 3 shows six sets of retarding potential sweeps from the RIMS radial analyzer at or before the first $-8 \mathrm{~V}$ 


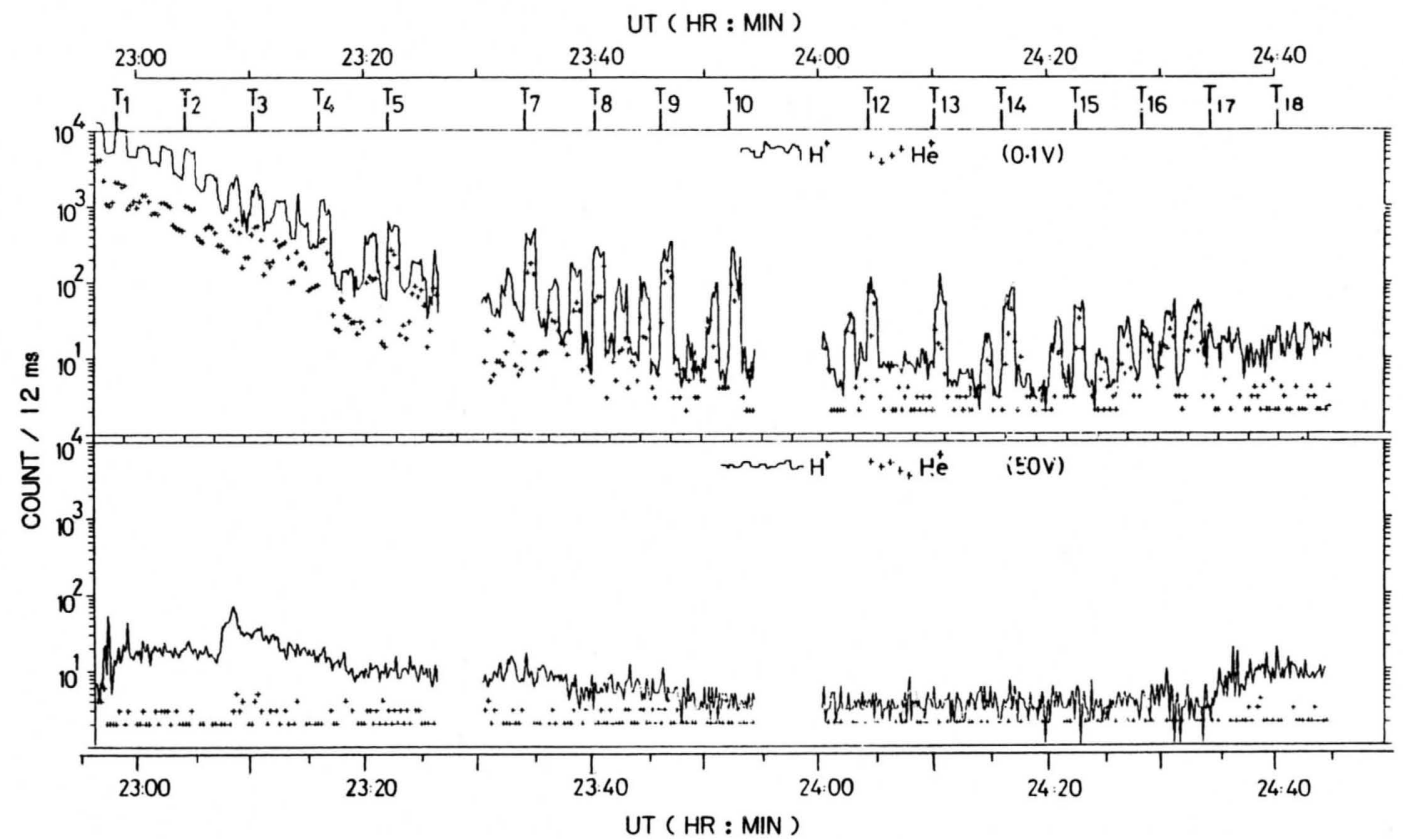

Fig. 2. The RIMS H $\mathrm{H}^{+}$(solid line) and $\mathrm{He}^{+}$(crosses) count rate as a function of time for the period $2256 \mathrm{UT}$ on October 14 to 0045 UT on October 15. The upper panel shows data for a retarding potential of $0.1 \mathrm{~V}$, while the lower panel is for a retarding potential of $50 \mathrm{~V}$. The times at which the analyzer was in the $-8 \mathrm{~V}$ biased aperture mode are indicated by the arrows and are labeled $T_{1}$ to $T_{17}$.

aperture bias phase $\left(T_{1}\right)$ and, consequently, in the region described above as the outer plasmasphere. The $\mathrm{H}^{+}$and $\mathrm{He}^{+}$average RPA spectra are shown in the upper and lower panels, respectively. Each sweep is the average of all samples with $\pm 10^{\circ}$ of the phase angle stated and consists of count rates of 32 energies spaced at logarithmi- cally equal voltage intervals from 0 to $51 \mathrm{~V}$. The three panels correspond to data taken under different aperture bias voltages; $0,-4$, and $-8 \mathrm{~V}$ for the left, center, and right panels, respectively. In each panel a thermal plasma (TP), which appears to be drifting owing to the spacecraft velocity, is present; however, it is only for the nonzero

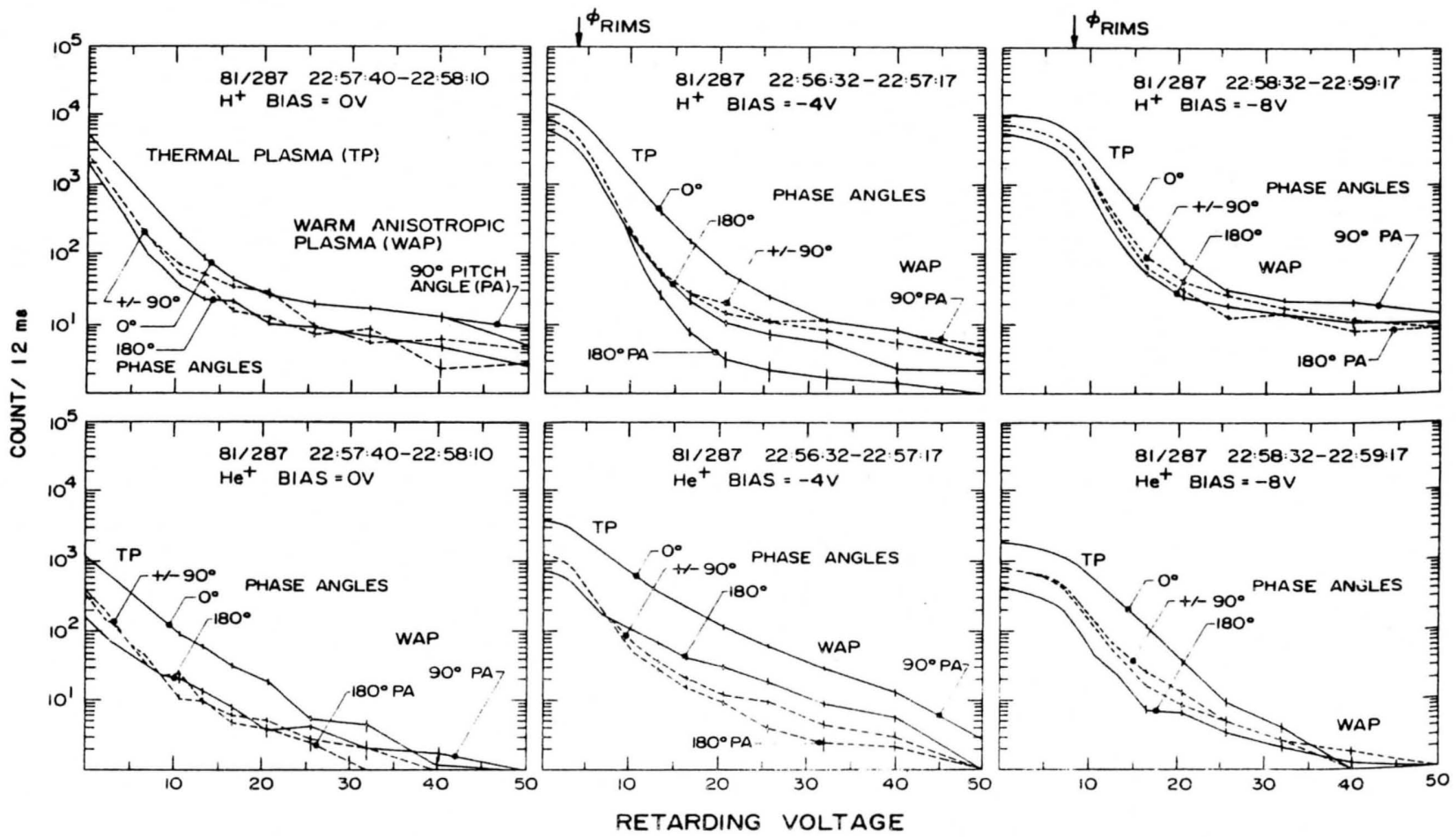

Fig. 3. $\mathrm{H}^{+}$(upper panels) and $\mathrm{He}^{+}$(lower panels) retarding potential sweeps from the radial analyzer for three different aperture bias voltages: $0 \mathrm{~V},-4 \mathrm{~V}$, and $-8 \mathrm{~V}$, respectively, for the left, center, and right panels. Each panel consists of 13 successive sweeps that covers one spin of the spacecraft. All the data are from the region defined as the outer plasmasphere. 
aperture bias voltages that the full retarding characteristic is observed. This characteristic, identified by $\Phi_{\text {RIMS }}$ on the central and right panels of Figure 3, is the retarding voltage that balances the combined spacecraft and RIMS aperture bias. The quantity $\Phi_{\text {RIMS }}$ is identified as the intersection of the approximately constant count rate region and the extrapolation of a straight line fit to the linearly decreasing count rate region of the RIMS retarding characteristic. Without a knowledge of $\Phi_{\text {RIMS }}$ it would be exceedingly difficult to infer the ion density. As will be shown below, the TP is relatively isotropic and has a $2 \mathrm{eV}$ temperature that is quite characteristic of the outer (hot) plasmasphere [Decreau et al., 1982; Comfort et al., 1982a]. At higher retarding voltages in Figure 3, the sweeps are flatter and appear to have an enhanced modulation. This is because the plasma is no longer isotropic and shows significantly depleted loss cones or trapped distributions at the higher energies $(\sim 30 \mathrm{eV})$. In the six panels of Figure 3 this region of warm anisotropic plasma is labelled WAP.

The analysis of such retarding potential sweeps and the associated spacecraft ram modulation has been extensively studied [Whipple, 1974; Singh and Baugher, 1981; Comfort et al. 1982a]. In this paper we wish to obtain the general characteristics of plasma composition $\left(\mathrm{H}^{+} / \mathrm{He}^{+}\right)$ temperature, density and anisotropy, hence we will carry out an analysis that allows for the zeroth-order spacecraft and instrument effects and describes the plasma by its deviation from isotropic Maxwellian populations. This procedure assumes a model wherein the RIMS count rate can be simulated by a superposition of several Maxwellian plasmas that are drifting relative to the spacecraft and are accelerated into the RIMS analyzer. The acceleration is due to a combination of spacecraft potential and aperture bias voltage. A detailed description of this technique is given by Sojka and Schunk [1982] and follows closely the ISEE satellite analysis procedure of D. T. Young (private communication, 1980). Although spacecraft sheath effects have been ignored in the modeling procedure this is not a serious limitation throughout the modeled region. There is also some uncertainty in the relative density calibrations of the DE/RIMS versus the plasma wave instrument [Comfort et al., 1982b]. Therefore the absolute densities reported here are subject to some uncertainty, but the relative densities of the various ions in the regions of interest are accurate.

In Figure 4 the model has been applied to the TP associated with the $-4 \mathrm{~V}$ aperture bias data of Figure 3 (central panels). The right and left panels of Figure 4 show the radial analyzer spin modulation for $\mathrm{H}^{+}$and $\mathrm{He}^{+}$, respectively. A spin angle of $0^{\circ}$ corresponds to the radial detector viewing along the spacecraft velocity vector. (The spin phase sense used throughout this paper is opposite to that used by the DE project team.) Data from 2256:41 UT to 2257:13 UT are shown for two different retarding voltages, $4.4 \mathrm{~V}$ associated with the TP and $26 \mathrm{~V}$ associated with the WAP. The solid line represents the modeled count rate at $4.4 \mathrm{~V}$ based on plasma and instrument parameters given in Table 2, 2256:41 UT to 2257:13 UT. For $\mathrm{H}^{+}$, left panel of Figure 4, good agreement is obtained between the model and the TP, while for $\mathrm{He}^{+}$, right panel of Figure 4, the agreement is good in the ram

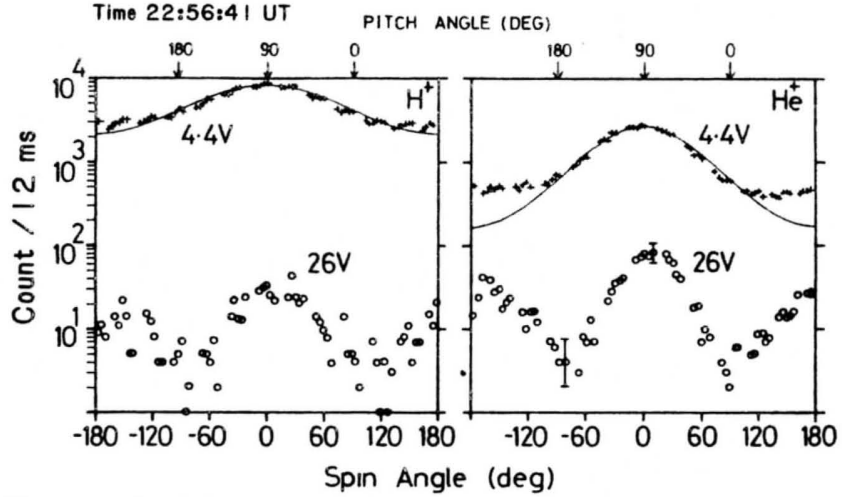

Fig. 4. $\mathrm{H}^{+}$(left panel) and $\mathrm{He}^{+}$(right panel) radial analyzer count rates at two retarding potentials, 4.4 and $26 \mathrm{~V}$, as a function of angle relative to the spacecraft velocity direction. The RIMS data are shown as crosses, while a model comparison is plotted as a solid line. The loss cone regions corresponding to pitch angles near $0^{\circ}$ and $180^{\circ}$ are identified by the pitch angle scale at the top of each panel. These data are from the outer plasmasphere, 2256:41 UT to 2257:13 UT.

direction but in the wake direction the count rate is a factor of 2 too high. Neither the $\mathrm{H}^{+}$nor $\mathrm{He}^{+}$WAP data show a ram modulation; in fact both show markedly depleted loss cones or trapped distributions (compare with the pitch angle scale at the top of Figure 4). The full depth of these loss cones is masked by the wide range of pitch angles $(\alpha)$ covered by the radial analyzer when viewing along the magnetic field line $\left(0<\alpha \lesssim 55^{\circ}\right)$. Table 2 contains the WAP characteristics based on fitting the count rates along the ram direction; consequently, the densities are only an upper limit. Comparing the WAP for $\mathrm{H}^{+}$and $\mathrm{He}^{+}$reveals that initially the effective $\mathrm{He}^{+}$density exceeds that of $\mathrm{H}^{+}$by a significant factor. However, by 2258:00 $\mathrm{UT} \mathrm{H}^{+}$again dominates. Over this same time interval the overall TP plasma density decreased from $530 \mathrm{~cm}^{-3}$ to $250 \mathrm{~cm}^{-3}$ with the $\mathrm{H}^{+} / \mathrm{He}^{+}$ratio remaining constant at about 2.7.

\subsection{Plasmapause}

The two component, TP and WAP, $\mathrm{H}^{+}$and $\mathrm{He}^{+}$plasma continues into the plasmapause region where the density decreases to about $10 \mathrm{~cm}^{-3}$. As the plasmapause is crossed, both the TP and WAP populations undergo modification. Figure 5 shows the spin modulation of $\mathrm{H}^{+}$ (left panel) and $\mathrm{He}^{+}$(right panel) for the radial analyzer at $T_{2}$ (2304:25 UT to 2304:41 UT). Also plotted are the appropriate model values (solid lines). In each panel the upper curve and data are for a retarding potential of $32 \mathrm{~V}$. The $\mathrm{H}^{+}$low-energy data and model agree well, while for the high-energy data a loss cone depletion is evident, as was the case for 2256:41 UT to 2257:13 UT of Figure 4. However, the loss cone is no longer as depleted as that shown in Figure 4, and yet the loss cone angle has decreased only from $13.8^{\circ}$ to $11.8^{\circ}$ in this interval. Therefore some plasma process must have been operative to make the WAP component more isotropic. For the $\mathrm{He}^{+}$ data and model (right panel of Figure 5) the agreement for the low-energy population is not good in the ram direction. Indeed, the data show a very flat peak over almost 


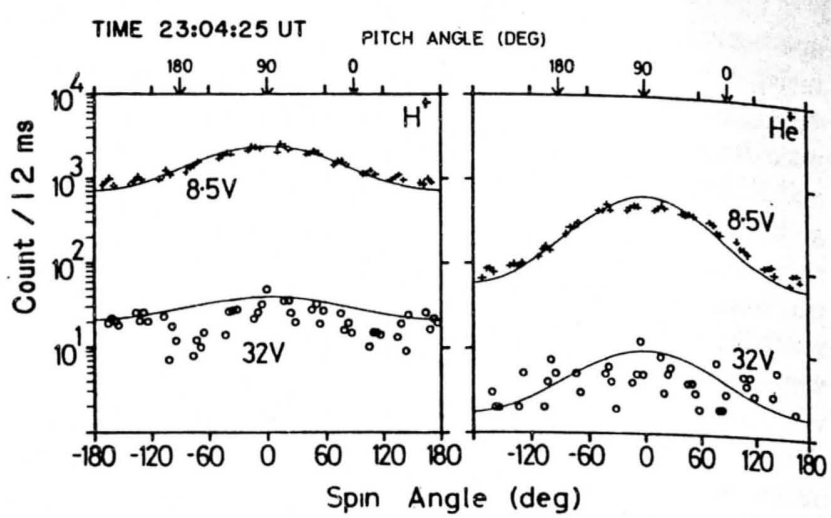

Fig. 5. $\mathrm{H}^{+}$(left panel) and $\mathrm{He}^{+}$(right panel) radial analyzer count rates at two retarding potentials, 8.5 and $32 \mathrm{~V}$, as a function of angle relative to the spacecraft velocity direction. The RIMS data are shown as crosses, while a model comparison is plotted as a solid line. These data are from the plasmapause 2304:25 UT to 2304:41 UT $\left(T_{2}\right)$.

$120^{\circ}$ of spin phase. This flattening results from the appearance of two thermal source cone populations at $0^{\circ}$ and $180^{\circ}$ pitch angle in addition to the isotropic thermal plasma. Further examples shown below as the spacecraft leaves the plasmapause region will adequately confirm this interpretation. Hence the thermal isotropic plasma component appears to become anisotropic as beams form along and opposite to the magnetic field direction.

Table 3 lists the plasma parameters for TP and WAP components. As can be seen, the density of the thermal component decreases, while that of the warm component increases. This increase is most marked between 2307:21 UT to 2307:45 UT, where a five-fold increase in the warm $\mathrm{H}^{+}$density is observed (see also lower panel of Figure 2). Figure 6 shows the temporal (spatial) increase in the count rate associated with the $+Z$ direction axial analyzer in the 2307:21 UT to 2307:45 UT. This analyzer always looks at $90^{\circ}$ to the ram direction and at a fixed pitch angle; hence it can be used to observe time variations of the order of a few seconds. Both $\mathrm{H}^{+}$(lower panel) and $\mathrm{He}^{+}$ (upper panel) show a correlated increase in count rate between 2307:30 UT to 2307:45 UT. The increase occurs over an interval of $15 \mathrm{~s}$, which, given that the spacecraft velocity is about $4.2 \mathrm{~km} / \mathrm{s}$ across ' $L$ ' shells, would correspond to a distance of $63 \mathrm{~km}$. This increase is largest for $\mathrm{H}^{+}$, a factor of 5 , while for $\mathrm{He}^{+}$the count rate increases by a factor of 3.5. At the same time the thermal plasma density shows a slight decline (see Table 3 ).

\subsection{Depleted Dayside Magnetosphere}

As the spacecraft moves to higher $L$ values, the densities decrease rapidly and the spacecraft potential becomes more positive. Table 4 shows the TP and WAP populations up until the time that the count rate becomes statistically uncertain (noise level). For $\mathrm{He}^{+}$this occurs at $T_{8}$, while for the major ion $\mathrm{H}^{+}$, it occurs at $T_{10}$. In going from an $L$ of 3.8 to 6 the density decreased from about $100 \mathrm{~cm}^{-3}$ to less than $0.50 \mathrm{~cm}^{-3}$, a decrease of more than 2 orders of magnitude. The temperature characteristics of these two populations remained about the same; however, for both $\mathrm{H}^{+}$and $\mathrm{He}^{+}$the WAP became almost isotropic. 


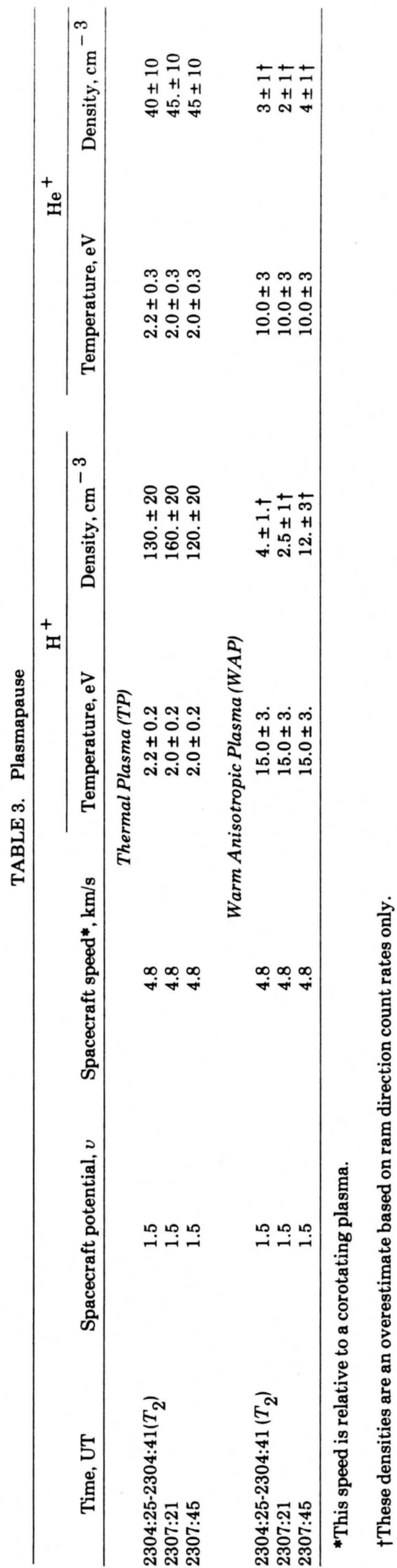

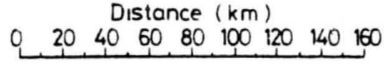
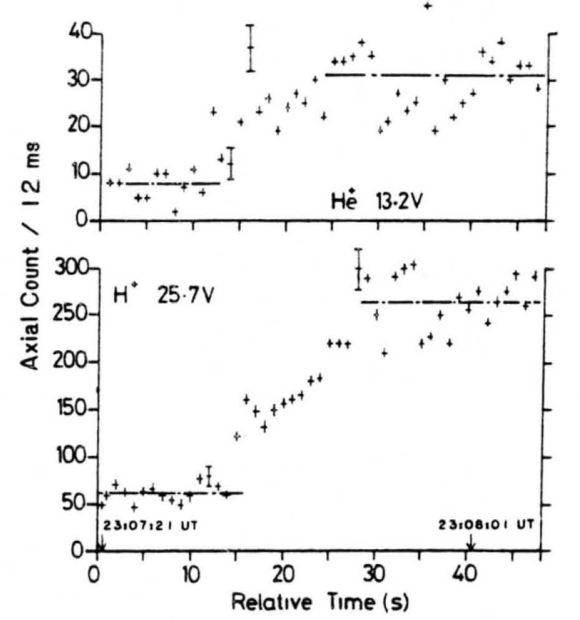

Fig. 6. Temporal evolution of the warm $\mathrm{H}^{+}$(bottom panel) and $\mathrm{He}^{+}$(top panel) plasma components at a retarding potential of 25.7 and $13.2 \mathrm{~V}$, respectively, for $\mathrm{H}^{+}$and $\mathrm{He}^{+}$. A "cross $L$ shell" spatial scale is shown above the upper panel. These data are from the $+Z$ axial RIMS analyzer.

While good statistics were available the composition appeared to remain constant with $\mathrm{H}^{+} / \mathrm{He}^{+} \sim 2$ to 3 for the TP component and $\mathrm{H}^{+} / \mathrm{He}^{+} \sim 2$ to 5 for the WAP component. As already pointed out in conjunction with the TP component, a second field-aligned component appears. Indeed, its appearance is due to the TP density decreasing to a point at which the thermal field-aligned (TFA) component can be observed. This is further verified by the relative constancy of the TFA across the depleted dayside magnetosphere.

Figure 7 shows the evolution of the TFA and TP for $\mathrm{H}^{+}$ (left column) and $\mathrm{He}^{+}$(right column) across the dayside magnetosphere. Each panel of Figure 7 displays the spin modulation of the radial analyzer at a retarding potential just greater than $\Phi_{\text {RIMS }}$. The ram direction is at $0^{\circ}$, which approximately corresponds to $90^{\circ}$ pitch angle, and spin angles of $-90^{\circ}$ and $90^{\circ}$ are, respectively, pitch angles of $180^{\circ}$ and $0^{\circ}$ (see Figures 4 and 6 ). Since the magnetic field lies almost within the spacecraft spin plane, the RIMS radial analyzer scans all pitch angles. The "peak" like populations (the TFA) do not lie exactly along $0^{\circ}$ and $180^{\circ}$ pitch angles due to the ions low energies being only slightly greater than the spacecraft velocity, and consequently, the angle of detection is moved toward the ram direction. Initially, at $T_{3}$ (top two panels of Figure 7) the TP component dominates and only a general indication of the TFA components is present, with the most marked signatures being present for $\mathrm{He}^{+}$. By $T_{5}$ the TP density has decreased by an order of magnitude, and the TFA components are clearly visible. However, for $\mathrm{He}^{+}$only the TFA component from the northern hemisphere (closest ionosphere) is present. By $T_{8}$ both $\mathrm{H}^{+}$and $\mathrm{He}^{+}$show only a northern hemisphere peak and an almost negligible TP component. The TFA decreases slightly at $T_{13}$ and even more by $T_{15}$ in the vicinity of the CUSP region. Table 5 gives the temperature and density obtained by fitting an isotropic Maxwellian to the field-aligned component (i.e., when the TFA count rate is greatest). This density has 

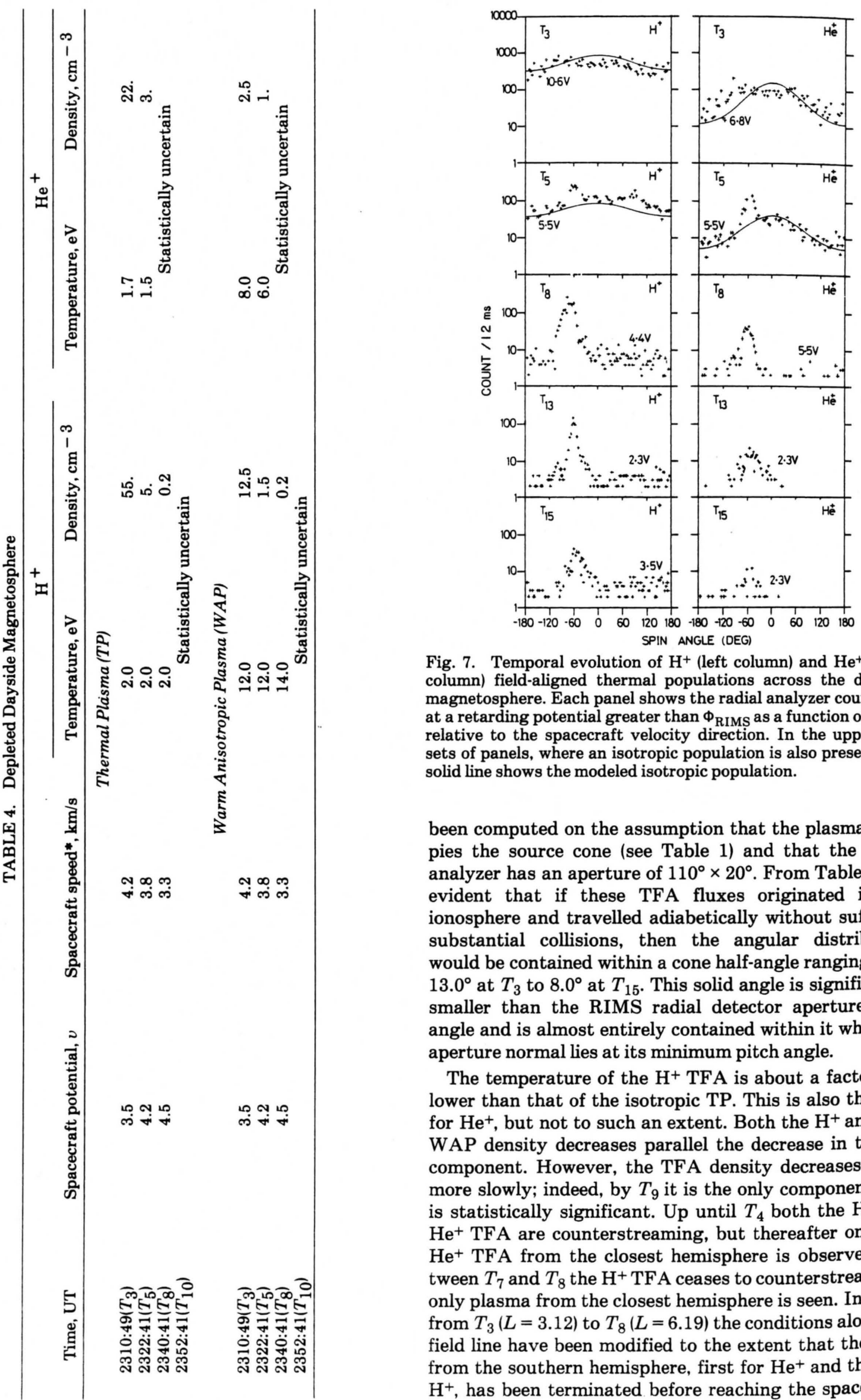

Fig. 7. Temporal evolution of $\mathrm{H}^{+}$(left column) and $\mathrm{He}^{+}$(right column) field-aligned thermal populations across the dayside magnetosphere. Each panel shows the radial analyzer count rate at a retarding potential greater than $\Phi_{R I M S}$ as a function of angle relative to the spacecraft velocity direction. In the upper two sets of panels, where an isotropic population is also present, the solid line shows the modeled isotropic population.

been computed on the assumption that the plasma occupies the source cone (see Table 1) and that the radial analyzer has an aperture of $110^{\circ} \times 20^{\circ}$. From Table 1 it is evident that if these TFA fluxes originated in the ionosphere and travelled adiabetically without suffering substantial collisions, then the angular distribution would be contained within a cone half-angle ranging from $13.0^{\circ}$ at $T_{3}$ to $8.0^{\circ}$ at $T_{15}$. This solid angle is significantly smaller than the RIMS radial detector aperture solid angle and is almost entirely contained within it when the aperture normal lies at its minimum pitch angle.

The temperature of the $\mathrm{H}^{+}$TFA is about a factor of 2 lower than that of the isotropic TP. This is also the case for $\mathrm{He}^{+}$, but not to such an extent. Both the $\mathrm{H}^{+}$and $\mathrm{He}^{+}$ WAP density decreases parallel the decrease in the TP component. However, the TFA density decreases much more slowly; indeed, by $T_{9}$ it is the only component that is statistically significant. Up until $T_{4}$ both the $\mathrm{H}^{+}$and $\mathrm{He}^{+}$TFA are counterstreaming, but thereafter only the $\mathrm{He}^{+}$TFA from the closest hemisphere is observed. Between $T_{7}$ and $T_{8}$ the $\mathrm{H}^{+}$TFA ceases to counterstream and only plasma from the closest hemisphere is seen. In going from $T_{3}(L=3.12)$ to $T_{8}(L=6.19)$ the conditions along the field line have been modified to the extent that the TFA from the southern hemisphere, first for $\mathrm{He}^{+}$and then for $\mathrm{H}^{+}$, has been terminated before reaching the spacecraft. 
Over this region the field line path length has increased by only about $40 \%$, whereas the plasma density has decreased by almost two orders of magnitude.

\subsection{Dayside Cusp}

Beyond $T_{15}(L=11.5)$ the plasma quickly changes its form (see Figure 1). Between $T_{15}$ and $T_{17}$ the $\mathrm{H}^{+}$fieldaligned component coming from the northern ionosphere is heated from $1 \mathrm{eV}$ to about $10 \mathrm{eV}$. However, $\mathrm{He}^{+}$ remains at around the noise level count rate. Beyond $T_{17}$ the $\mathrm{H}^{+}$temperature increases to such a point that by $T_{18}$ it is greater than about $30 \mathrm{eV}$ and $\mathrm{H}^{+}$is no longer retarded by the 0 to $51 \mathrm{~V}$ RIMS range. This warm plasma streaming out of the ionosphere is associated with the cusp region. The data display quite a systematic $L$ variation, showing how the hot cusp plasma replaces the much cooler closed field line plasma. Generally, the cusp plasma is only "weakly" field aligned.

\section{IONOSPHERIC PLASMA OUTFLOW}

\subsection{Field-Aligned Flow Speed}

In the initial analysis we assumed that the plasma had a Maxwellian distribution and that the only plasma drift velocities present were due to the motion of the spacecraft through the plasma. However, this type of analysis cannot tell us anything about the subsonic/supersonic character of the TFA ion distributions. As mentioned in the introduction, the theories describing the plasma outflow from the ionosphere can give either type of flow. Therefore it is of considerable interest to look at the TFA distributions in more detail in an effort to place limits on the flow speed.

The observed distribution is tightly collimated with respect to the pitch angle. The collimation is brought about by the divergence of the $\mathbf{B}$ field with altitude and the conservation of the first adiabetic invariant in a near collisionless plasma. Although the distribution started off in the collisionally dominated topside ionosphere (at a few thousand kilometers) as an isotropic drifting Maxwellian, the divergence of $\mathbf{B}$ has collimated the distribution by the time it reaches the DE- 1 altitude $(20,000 \mathrm{~km})$ to a source cone angle of about $10^{\circ}$, smaller than the resolution of the detector. Therefore detailed information on the two-dimensional distribution function cannot be obtained, but a one-dimensional energy analysis along the direction of the peak count rate can give us information on the temperature, field-aligned drift velocity and density of the TFA ions. Unfortunately, as we will see, this information is not unambiguous since the spacecraft potential is not accurately known. However, it is possible to obtain ranges of the temperature, field-aligned drift speed, and density for given values of the spacecraft potential.

In addition to the one-dimensional drifting Maxwellian energy analysis one can also obtain field-aligned flow velocity information from the observed pitch angle of the TFA peak flux. Since the spacecraft is traveling perpendicular to the $\mathbf{B}$ field the apparent pitch angle is simply the vector sum of the spacecraft velocity, the $\mathbf{E} \times \mathbf{B}$

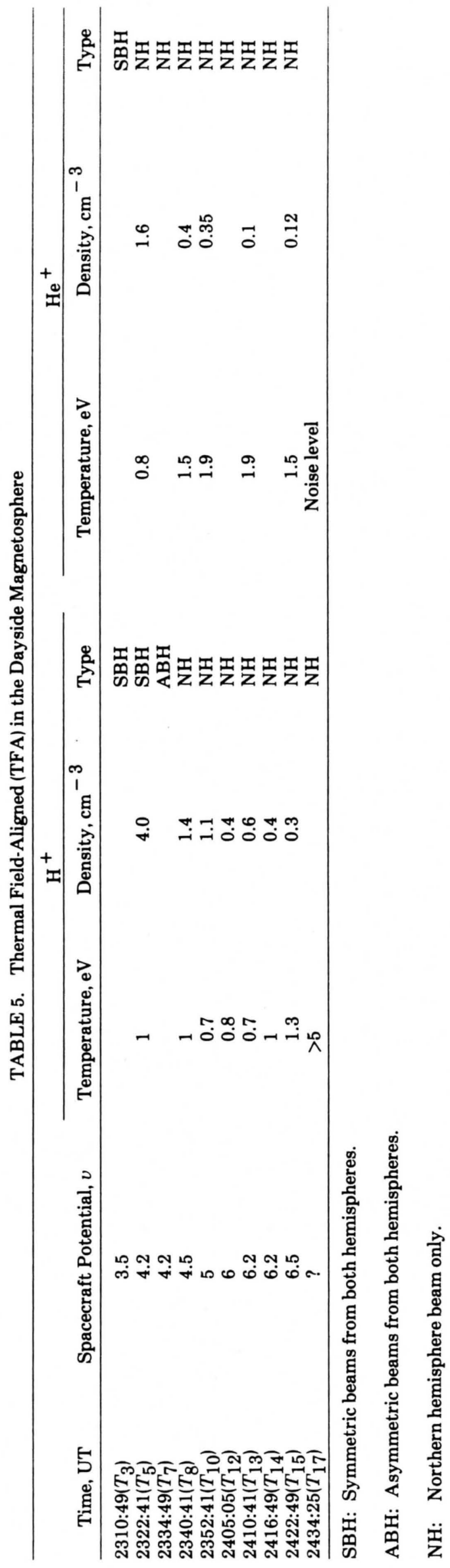


plasma drift velocity and the field-aligned flow velocity.

In this section, we use three different methods to try to characterize the TFA ion flows. First, the information on the apparent spin phase or pitch angle of the TFAs is used to estimate the effects of a field-aligned velocity. This is followed by a detailed parametric study using the one-dimensional drifting Maxwellian energy analysis. Finally, we compare the fluxes calculated from the energy analysis to the measured fluxes and try to determine the Mach number of the observed TFAs.

Figure 8 (top panel) shows the satellite geometry at $T_{5}$ for the $\mathrm{H}^{+}$and $\mathrm{He}^{+}$TFA components (see Table 5). The spacecraft is moving perpendicular to the magnetic field with a velocity of $3.8 \mathrm{~km} / \mathrm{s}$. Given that the $\mathrm{H}^{+}$and $\mathrm{He}^{+}$ temperatures are 1 and $0.8 \mathrm{eV}$, respectively, the corresponding thermal speeds are 13 and $6 \mathrm{~km} / \mathrm{s}$. Assuming initially that the plasma is neither corotating nor drifting under an $\mathbf{E} \times \mathbf{B}$ force, Figure 8 (upper panel) indicates that the two $\mathrm{H}^{+}$peaks should occur at $16^{\circ}$ from the magnetic field line, a total separation of $148^{\circ}$ from peak to peak through the ram direction. Also, the single $\mathrm{He}^{+}$peak should be further displaced by $16^{\circ}$ from the $\mathrm{H}^{+}$peak towards the ram direction. These angles are all assumed to be rotational angles in the satellite spin plane based on the assumption that the magnetic field is in this plane. Even if the magnetic field lines were not quite in this plane, the $55^{\circ}$ aperture perpendicular to the spin plane would ensure that the peak was detected. Figure 7 (second top left panel) shows that the observed separation between the two $\mathrm{H}^{+}$TFA peaks is about $150^{\circ}$ at $T_{5}$ and that the $\mathrm{He}^{+}$peak is further displaced by about $15^{\circ}$ from the $\mathrm{H}^{+}$peak towards the ram direction. Hence the assumption that the distribution are one-dimensional nondrifting Maxwellians along the direction of the peak flux for both $\mathrm{H}^{+}$and $\mathrm{He}^{+}$produces results that are somewhat consistent with the data.

However, if we now consider the one-dimensional energy analysis, the retarding characteristics for the TFA do not agree perfectly with a pure Maxwellian. Indeed, better agreement is obtained if a drift along the direction of observation is included. However, Whipple et al. [1974] have shown that the effects of particle fluxes passing through the surrounding satellite sheath can alter the resulting RPA characteristic in a manner somewhat similar to that observed here. At this stage it is not possible to determine whether or not such an effect for RIMS with its biased aperture would yield the observed characteristics. Therefore we will proceed by assuming that the TFA has a field-aligned drift.

Figure 9 shows the $T_{5}$ retarding potential sweep along the peak count spin angle for $\mathrm{H}^{+}$. Also shown are the model fits for a nondrifting Maxwellian and several drifting Maxwellians. Clearly the drifting Maxwellians produce a more favorable agreement with the data, especially the $25 \mathrm{~km} / \mathrm{s}$ case. The general trend indicates that as the parallel speed increases, the required model temperature and density of the Maxwellian decreases, while the required spacecraft potential increases. To some extent the large drift speeds can be ruled out because the corresponding spacecraft potentials are too large. Such large potentials are inconsistent with the spacecraft potential deduced from the isotropic TP population. In the iso-

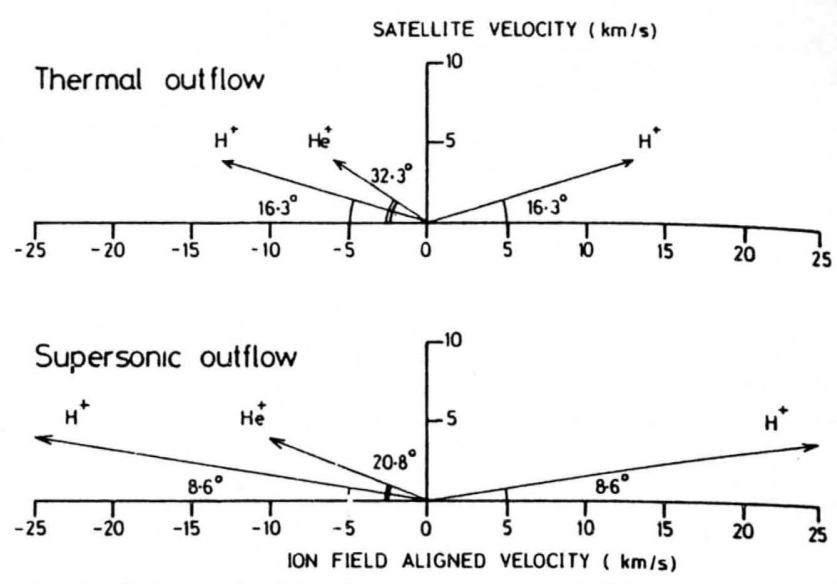

Fig. 8. Spin angle detection geometry for field aligned $\mathrm{H}^{+}$and $\mathrm{He}^{+}$beams at $T_{5}$. The upper panel is for only thermal outflow, while the lower panel is for a supersonic outflow velocity of 25 $\mathrm{km} / \mathrm{s}$ for $\mathrm{H}^{+}$and $10 \mathrm{~km} / \mathrm{s}$ for $\mathrm{He}^{+}$.

tropic case, the error or shift in the spacecraft potential over that deduced at $90^{\circ}$ to the magnetic field (ram direction) could not have been associated with such a large drift velocity.

From Figure 9 and the preceding discussion, a drift of up to about $30 \mathrm{~km} / \mathrm{s}$ could conceivably be consistent with the $\mathrm{H}^{+}$retarding characteristics. For $\mathrm{He}^{+}$a smaller drift, $12 \mathrm{~km} / \mathrm{s}$, could be possible. These drifts, however, together with only the spacecraft ram velocity would be inconsistent with the angular peak information. The lower panel of Figure 8 shows the parallel flow geometry at $T_{5}$ for the above parallel drifts. These angles are quite distinguishable from those obtained earlier for nondrifting Maxwellians. If the perpendicular speed could be increased to $6.5 \mathrm{~km} / \mathrm{s}$ from the ram speed of $3.8 \mathrm{~km} / \mathrm{s}$, reasonable angular agreement would be obtained. Such a drift speed would require an "E $\mathbf{x}$ B" drift velocity between $2.7 \mathrm{~km} / \mathrm{s}$ and $5.3 \mathrm{~km} / \mathrm{s}$, depending on the orientation of the $\mathbf{E}$ field relative to the ram direction. From Table 1 the magnetic field strength at $T_{5}$ is $0.01 \mathrm{G}$, which would then require an electric field between $2.7 \mathrm{mV} / \mathrm{m}$ and $5.3 \mathrm{mV} / \mathrm{m}$ to produce the required " $\mathbf{E} \times \mathbf{B}$ " speed. Such an electric field is not unreasonable. However, it is somewhat large for a magnetospheric dC field and opposite to the generally accepted convection direction in this region of the magnetosphere.

The lower temperature associated with the high drift velocities are still consistent with an ionospheric origin. Thus the current analysis does not rule out supersonic flow speeds up to about $30 \mathrm{~km} / \mathrm{s}$ for $\mathrm{H}^{+}$with plasma temperatures of the order of $0.2 \mathrm{eV}$. However, this flow speed can be regarded as an upper limit in that the associated spacecraft potential begins to diverge from that deduced from the isotropic plasma component observed at the same time. The existence of magnetospheric electric fields inside the plasmapause during active conditions is highly probable [Maynard et al., 1983], and therefore, our analysis indicates that a fieldaligned drift was probably present at $T_{5}$.

We have performed a similar analysis to that shown in Figure 9 for the data taken at time $T_{8}$ (2340 UT), which in two ways is a somewhat simpler case. First, the isotropic 
thermal plasma background is not present, and second, the plasma flow is only observed from one hemisphere. For these reasons the $T_{8}$ data probably better represents observations of the predicted polar wind than do those at $T_{5}$. It will be seen in Figure 10 that even here it is not possible to uniquely define the character of the observed plasma distribution, with either subsonic $(0.7 \mathrm{eV}$ plasma drifting at $7.5 \mathrm{~km} / \mathrm{s})$ or supersonic $(0.39 \mathrm{eV}$ plasma drifting at $17.5 \mathrm{~km} / \mathrm{s}$ ) flows being possible for plausible spacecraft potentials. However, the calculations of the flow velocity using the deflection of the peak from the magnetic field direction due to the spacecraft velocity flow favors higher flow velocities $(\sim 21 \mathrm{~km} / \mathrm{s})$ in this example.

\subsection{Ionospheric Plasma Escape Flux}

In the region poleward of $T_{5}$ for $\mathrm{He}^{+}$and $T_{8}$ for $\mathrm{H}^{+}$, a net ion outflow from the northern hemisphere is observed. To calculate the escape flux, it is only necessary to know the density, parallel velocity, and range of pitch angles over which the plasma is flowing; we will call this method 1. Unfortunately, as noted in the previous section, the parallel drift velocity is not well determined, ranging from 0 to $30 \mathrm{~km} / \mathrm{s}$ for $\mathrm{H}^{+}$and 0 to $12 \mathrm{~km} / \mathrm{s}$ for $\mathrm{He}^{+}$. As the drift velocity increases, the deduced density and spacecraft potential change, as does the escape flux. Hence, at $T_{5}$ the upward $\mathrm{H}^{+}$flux ranges from $1.0 \times 10^{7}$ $\mathrm{cm}^{-2} \mathrm{~s}^{-1}$ to $0.98 \times 10^{5} \mathrm{~cm}^{-2} \mathrm{~s}^{-1}$ for the nonstreaming and supersonic cases, respectively, assuming the plasma is contained within a source cone referenced to an ionospheric altitude of $2000 \mathrm{~km}$. These two fluxes correspond to fluxes of $2.6 \times 10^{8} \mathrm{~cm}^{-2} \mathrm{~s}^{-1}$ and $2.5 \times 10^{7} \mathrm{~cm}^{-2} \mathrm{~s}^{-1}$ at $2000 \mathrm{~km}$ if a constant flux is assumed to flow along the flux tube between $2000 \mathrm{~km}$ and the satellite. In deriving a flux from the RIMS retarding sweep, an increased out-

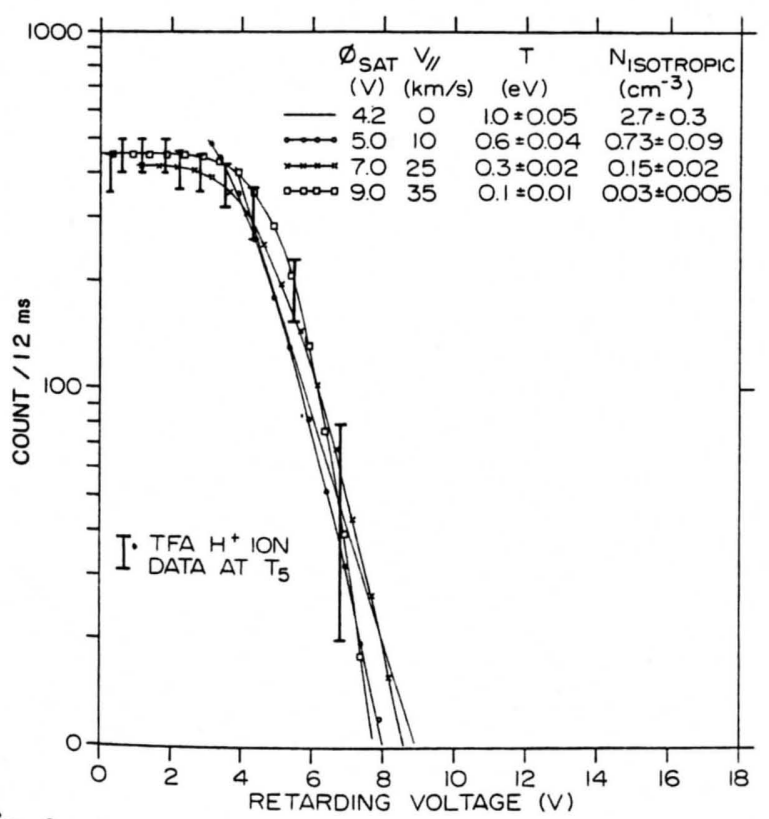

Fig. 9. A comparison of various model retarding voltage sweeps with a data set of field-aligned $\mathrm{H}^{+}$ions taken at $T_{5}$. The isotropic background has been subtracted from the field-aligned data set. Model values with field-aligned drift velocities ranging from 0 to $35 \mathrm{~km} / \mathrm{s}$ are shown.

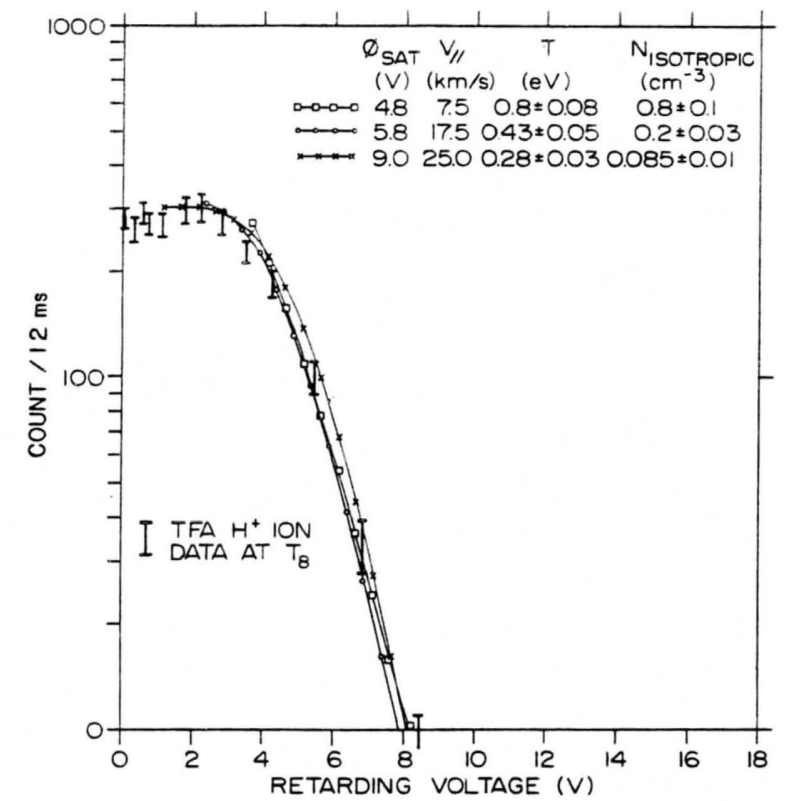

Fig. 10. A comparison of various model retarding voltage sweeps with a data set of field-aligned $\mathrm{H}^{+}$ions taken at $T_{8}$. Model values with field-aligned drift velocities ranging from 0 to $30 \mathrm{~km} / \mathrm{s}$ are shown.

flow speed is associated with a reduced escape flux, owing to the reduced density inferred from the analysis. Therefore, a wide range of fluxes are deduced because of the large uncertainty in outflow speed.

An alternative method (method 2) that bypasses the density and velocity calculation can be applied to the data. As already noted, at the satellite altitude the source cone half-angle ranges from $13^{\circ}$ to $9^{\circ}$ over the region where outflowing plasma is observed (see Table 1). This angular cone can almost be entirely contained within the $\pm 55^{\circ} \times \pm 10^{\circ}$ radial analyzer aperture. Hence if the TFA plasma is the only ion plasma component present, then the count rate looking along the beam direction (peak count) is the total number of ions crossing an area equal to the effective analyzer aperture area. By choosing the negative aperture bias voltage to be equal to or greater than spacecraft voltage, most of the ions in the TFA plasma are able to enter the analyzer. Table 6 shows a comparison between the escape flux inferred from method 1 and that described above using method 2 for both $\mathrm{H}^{+}$ and $\mathrm{He}^{+}$. The escape flux at $2000 \mathrm{~km}$ is also shown for method 2 in Table 6. A comparison of the two techniques indicates that on the average the inferred flux (method 1) is about $50 \%$ greater than the direct flux (method 2). If a field-aligned drift velocity is introduced into the inferred flux calculation, a lower flux results. Hence the comparison of the two techniques favors a nonzero fieldaligned drift velocity $\left(\sim 7 \mathrm{~km} / \mathrm{s}\right.$ for $\mathrm{He}^{+}$and $\sim 20 \mathrm{~km} / \mathrm{s}$ for $\mathrm{H}^{+}$). However, as before, the uncertainty in the flux determination will allow drift velocities for $\mathrm{H}^{+}$from $\sim 0$ to 25 $\mathrm{km} / \mathrm{s}$ and for $\mathrm{He}^{+}$between 0 and $10 \mathrm{~km} / \mathrm{s}$.

\subsection{Plasmaspheric Refilling}

Although plasma measurements were made for $L>3$, no net ionospheric escape flux was evident until $L=6.2$ 


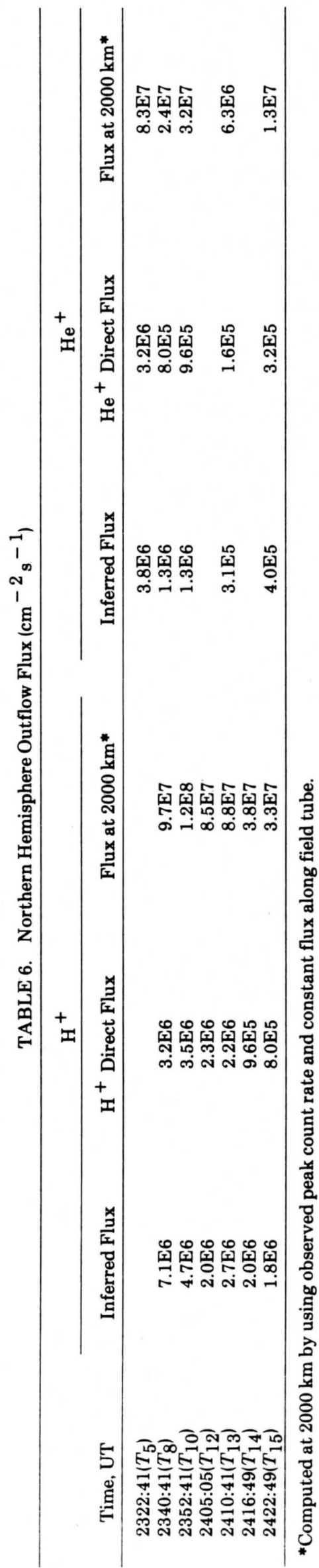

for $\mathrm{H}^{+}$and $L=4.7$ for $\mathrm{He}^{+}$. Prior to these two $L$ shells the plasma had no observable net flow, since it was either dominated by an isotropic component or contained counterstreaming beams of comparable magnitude. Beyond these two regions, only plasma streaming from the closest ionosphere was observed.

If we assume symmetry of flow between the northern and southern ends of the magnetic field lines, then the observed fluxes on high $L$ shells represent half the flux entering into, and remaining within, the flux tube above the spacecraft. This assumption should be reasonable, since the data were acquired near equinox. However, at the time of the observations the magnetic geometry was not symmetric (see Figure 1). As plasma collects at the equator, the whole flux tube corotates from dawn to dusk and probably also drifts sunward, owing to a magnetospheric E x B drift. The purely corotating plasma takes half a day to drift from dawn to dusk, while at the plasmapause the plasma will take longer since it tends to stagnate as it approaches dusk. Flux tubes beyond the plasmapause will not necessarily reach dusk, since the $\mathbf{E}$ x B drift causes them to drift to the dayside magnetopause. Nevertheless, in order to estimate how much of the flux tube is refilled, a half-day will be used as the time period over which refilling is carried out.

Table 7 shows the average density increase in a flux tube over half a day if we assume uniform filling of the flux tube. The plasma probably does not fill the flux tube uniformly, but preferentially accumulates near the equator. Hence, these average values are too high for low altitude regions and slightly too low in the equatorial regions. For $\mathrm{H}^{+}$, the rate of ionospheric loss to the magnetosphere beyond $L=6.2$ starts at about $12.2 \mathrm{~cm}^{-3}$ per half day and decreases to $2.8 \mathrm{~cm}^{-3}$ per half day at $L=$ 9.6. At the higher $L$ values, the plasma no longer reaches dusk but will be carried into the dayside magnetopause and will provide a relatively constant source of lowenergy plasma for the magnetosheath and possible return to the magnetosphere. At $L$ values less than $6.2, \mathrm{H}^{+}$is observed to counterstream in a more dense background plasma; however, direct refilling via the beams is apparently not occurring. For $\mathrm{He}^{+}$the inner $L$ shell at which refilling is observed is at 4.7, significantly further inside the dayside magnetosphere than for $\mathrm{H}^{+}$. A consequence of this lower $L$ value extent of the $\mathrm{He}^{+}$refilling is that the equatorial $\mathrm{H}^{+} / \mathrm{He}^{+}$composition ratio is quite independent of the composition ratio of the ion beams and of the ionospheric source. The observations would indicate that the equatorial outer plasmasphere regions are relatively rich in the heavier $\mathrm{He}^{+}$ion. As shown in Table 7, the average $\mathrm{He}^{+}$density refilling rates are smaller, yet comparable to, those of $\mathrm{H}^{+}$.

From Figure 1 it is evident that DE- 1 had cut the refilling field tubes approximately halfway between the ionosphere and the equator for the lower $L$ values and nearer the ionosphere for the higher $L$ values. Although there is an indication of a buildup in nonfield aligned plasma density at $L$ values less than $\sim 4$, there is no clear signature for a corresponding increase at higher $L$ values, indicating that refilling apparently begins at the equator. A consequence of this equator refilling would be that density gradients associated with the plasmapause are 


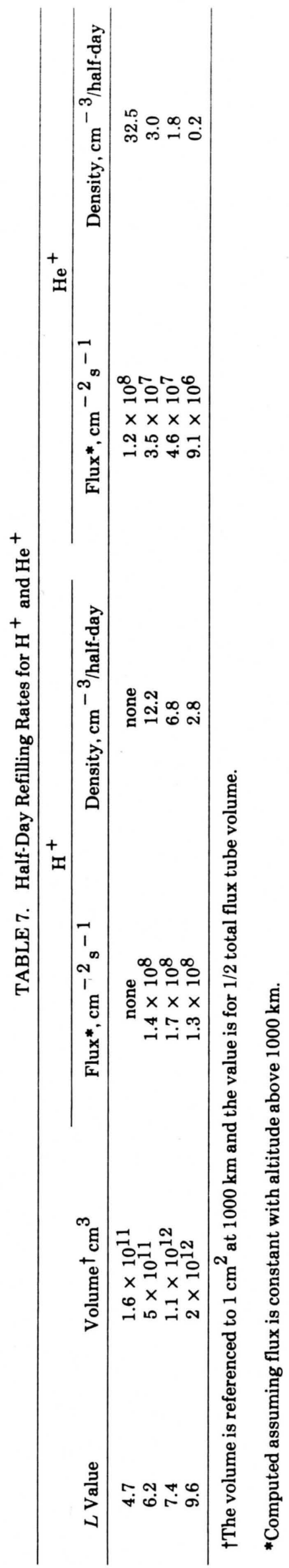

altitude or latitude dependent [Foster et al., 1978]. Also, since the plasma builds up with time, the dusk densities will be higher than at dawn if the flux tube geometry does not drastically change as it drifts from dawn to dusk.

There are some interesting aspects of refilling that are suggested by the observations. If a two-stream instability is responsible for the accumulation of plasma near the equator, then the fact that the $\mathrm{H}^{+}$flow is unidirectional only outside of $L=6.2$ (whereas field-aligned $\mathrm{H}^{+}$flows began near $L=4.2$ ) may suggest that even small densities of warm isotropic plasma observed in the region may act to stabilize the refilling instabilities; hence the later stages of refilling would have to proceed by a different mechanism. Furthermore, the fact that the $\mathrm{He}^{+}$unidirectional flow begins near $L=4.7$ suggests a mass selective wave-particle trapping of $\mathrm{He}^{+}$in the outer plasmasphere. Certainly, the process of refilling appears to be much more complicated than suggested by early theories.

\subsection{Wave-Particle Phenomena}

It has become clear in recent years (see Rycroft and Lemaire [1975] and references therein) that a great variety of plasma waves are associated with the plasmapause region. In addition, some direct correlations of waves and particles have been made. Although direct wave-particle correlations appear more common for the suprathermal electrons, these events still represent significant exchanges of energy between the waves and plasma, and waves also have been associated with the ion cyclotron frequencies. Thus it may only be possible to understand fully the filling process if the necessary wave-particle interactions are taken into account. We must ask first what aspects of the population are suggestive of waveparticle interactions and second what sort of wave modes are likely to be involved. Clearly, the electron distribution function may also be important (it is not available at low energies $(\leqslant 5 \mathrm{eV})$ on DE-1), since energy is clearly entering into the region of interest via ionospheric photoelectrons, as observed by the GEOS 1 spacecraft (G. L. Wrenn, private communication, 1982).

From the observed particle distributions, there are at least three distinct populations that are likely candidates for wave-particle interactions; these are (1) the outer plasmasphere 1 to $2 \mathrm{eV}$ population (TP), (2) the warm 5 to $20 \mathrm{eV}$ anisotropic population (WAP), and (3) the cold ions escaping into the depleted dayside magnetosphere (TFA). Populations (1) and (2) are much hotter than the ionospheric plasma, where typical temperatures remain below $0.5 \mathrm{eV}$. Hence although the ions are of ionospheric origin they have experienced an additional energization. The 1 to $2 \mathrm{eV}$ plasma is quite typical of the warmer region just outside the plasmasphere, as observed by Bezrukikh and Gringauz [1976] and Horwitz and Chappell [1979], and is a normal part of the plasmasphere. Because the population is isotropic, no obvious source region along the flux tube can be identified, and hence heating could occur all along the field line. The plasma is apparently collisionless since at the plasmapause counterstreaming lower energy beams are observed (see Figure 7, upper two sets of panels). In these panels the solid line represents an isotropic plasma and the deviations from this curve repre- 
sent the field-aligned lower energy plasma. Over this region, identified as period $T_{3}$ to $T_{5}$, the density decreases from $100 \mathrm{~cm}^{-3}$ to $10 \mathrm{~cm}^{-3}$. Beyond $T_{5}$ the density further decreases and for $\mathrm{H}^{+}$the counterstreaming ceases and only the northern hemisphere (closest-ionosphere) beam is observed. One explanation is that the disappearance of the southern hemisphere beam is indicative of a trapping or thermalization of the beams at an altitude higher than the satellite. Near the equator, the background isotropic plasma density has decreased below a threshold value at which the two counterstreaming beams can interact to modify these distributions to the extent that they do not leave this region as a field-aligned cold plasma.

Population (2) is the hottest of the three, with temperatures in the $5 \mathrm{eV}$ to $20 \mathrm{eV}$ range and in addition is highly anisotropic. From Figure 4 (the higher energy (26 V) data set) it is apparent that marked loss cones are present for both $\mathrm{H}^{+}$and $\mathrm{He}^{+}$. These loss cones are significantly larger than the local spacecraft $14.8^{\circ}$ source cone angle associated with the ionosphere at $2000 \mathrm{~km}$. Over the period $T_{3}$ to $T_{8}$, as this population diminishes, the loss cones fill up and by $T_{8}$ the population is almost isotropic. Clearly, since an extended loss cone is present the warm plasma could not be heated at low altitudes. In addition, since the bulk of the population has a maximum energy perpendicular to the magnetic field, perpendicular heating must occur in the source region.

\section{SUMMARY AND DISCUSSION}

Using data acquired on October 14-15, 1981, by the RIMS instrument on the DE-1 satellite, we have observed three ion populations whose temperatures were less than $20 \mathrm{eV}$ in the dayside magnetosphere (see Figure 11). These three populations, although of ionospheric origin, have undergone quite different evolutions and have the following general characteristics:

1. The thermal plasma (TP) population is isotropic and has a temperature between 1.5 and $3 \mathrm{eV}$ and is the main constituent of the outer plasmasphereplasmapause. The $\mathrm{H}^{+} / \mathrm{He}^{+}$density ratio lies between 2 and 4.

2. The warm anisotropic plasma (WAP) population displays greatly depleted and extended loss cones with temperatures ranging from 5 to $20 \mathrm{eV}$ and is observed in the plasmapause region. The $\mathrm{He}^{+}$temperatures are typically $50 \%$ less than the $\mathrm{H}^{+}$temperatures, while the $\mathrm{H}^{+} / \mathrm{He}^{+}$density ratio ranges from 0.1 to 6 .

3. The thermal field-aligned (TFA) population consists of counterstreaming beams of both ionospheric $\mathrm{H}^{+}$and $\mathrm{He}^{+}$in the vicinity of the plasmapause, beyond this region only ions from the closest ionosphere are observed. These beams have temperatures $\leqslant 1.5 \mathrm{eV}$ and $\leqslant 2.0 \mathrm{eV}$ for $\mathrm{H}^{+}$and $\mathrm{He}^{+}$, respectively, and respective parallel drift velocities of $<30 \mathrm{~km} / \mathrm{s}$ and $<15 \mathrm{~km} / \mathrm{s}$. The $\mathrm{H}^{+} / \mathrm{He}^{+}$flux ratio ranges from 3 to 10 .

The third of these populations is the source of plasma for the initial stages of a flux tube refilling process, which has the following properties:

1. Beyond $L=4.7$ for $\mathrm{He}^{+}$and $L=6.2$ for $\mathrm{H}^{+}$, beams filling the loss cone appear to interact and accumulate in the equatorial regions of the flux tubes.

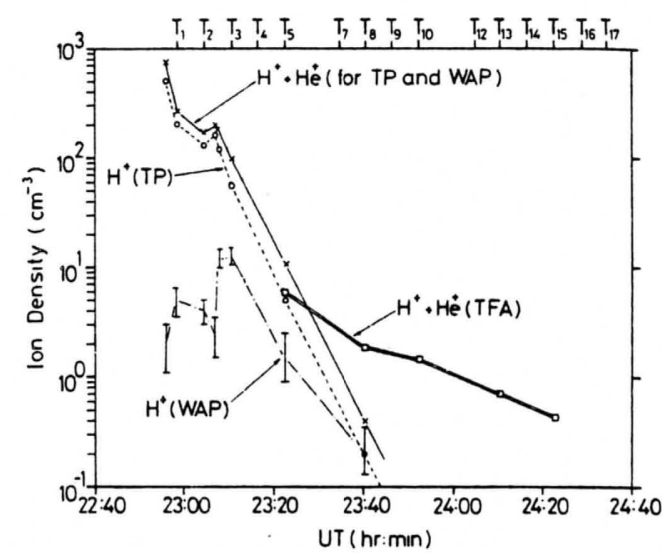

Fig. 11. Computed local density variation as DE-A crossed the dayside magnetosphere (see Figure 1). The density variation is shown for each of the three plasma types: thermal plasma (TP), warm anisotropic plasma (WAP), and thermal field-aligned plasma (TFA). In the case of the TFA the density computation includes corrections for pitch angle range and aperture size. Fine structure is indicated between $T_{2}$ and $T_{3}$, other fine structure may exist between the other $-8 \mathrm{~V}$ bias observations labeled as $T_{1}$ to $T_{17}$.

2. Filling rates diminish with increasing latitude from 12 to $3 \mathrm{~cm}^{-3} /$ half day for $\mathrm{H}^{+}$over the range of $L=6.2$ to 9.6 and from 33 to $0.2 \mathrm{~cm}^{-3} /$ half day for $\mathrm{He}^{+}$over the range of $L=4.7$ to 9.6 .

3. At latitudes equatorward of $L=4.7$ for $\mathrm{He}^{+}$, and $L$ $=6.2$ for $\mathrm{H}^{+}$, these counterstreaming beams appear to penetrate each other without interaction.

4. Instruments on DE-1 are unable to determine unambiguously the drift characteristics of the refilling plasma, which could be either subsonic or supersonic.

In the subsections that follow, we briefly discuss both the polar wind and the plasmaspheric refilling process, with emphasis given to show how the DE-1 measurements relate to our current understanding of these piocesses.

\subsection{Polar Wind}

At the present time, the bulk of the information on the polar wind derives from theoretical models of a steady state ionospheric outflow [cf. Raitt and Schunk, 1983]. On the basis of these model studies, the plasma flowing up from the polar ionosphere into the magnetosphere should have the following characteristics:

1. The composition is dominated by the light ions $\mathrm{H}^{+}$ and $\mathrm{He}^{+}$,

2. At $1000 \mathrm{~km}$ the $\mathrm{H}^{+}$escape flux should vary from $10^{7}-5 \times 10^{8} \mathrm{~cm}^{-2} \mathrm{~s}^{-1}$ and the $\mathrm{He}^{+}$escape flux from $10^{5-10^{7}}$ $\mathrm{cm}^{-2} \mathrm{~s}^{-1}$, depending on the geophysical conditions,

3. The ion temperatures should be less than $8000^{\circ} \mathrm{K}$ (less than $1 \mathrm{eV}$ ),

4. In the collisionless region at high altitudes, the $\mathrm{H}^{+}$ temperature distribution should be anisotropic, with $T_{\|}$ $>T_{\perp}$ for supersonic $\mathrm{H}^{+}$outflow and $T_{\|}<T_{\perp}$ for subsonic $\mathrm{H}^{+}$outflow in combination with a downward $\mathrm{H}^{+}$heat flow,

5. In the collisionless region, the $\mathrm{H}^{+}$velocity distribution should be asymmetric, with an elongated tail along 


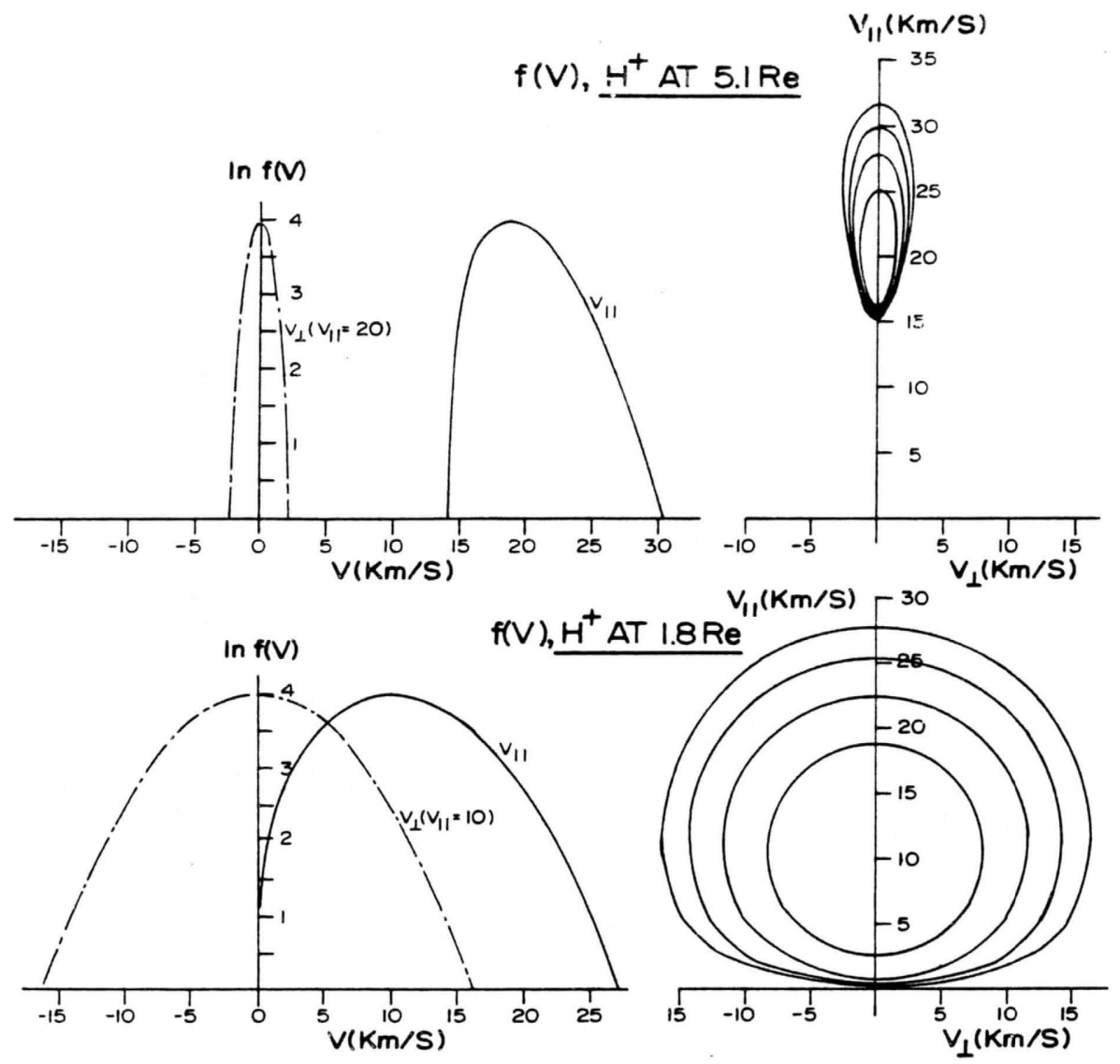

Fig. 12. Schematic diagram showing the kinetic evolution of the $\mathrm{H}^{+}$velocity distribution for a collisionless supersonic polar wind. Starting with a drifting Maxwellian at the exobase (lower panel), the distribution function at higher altitudes becomes asymmetric and anisotropic (upper panel) as the ions flow along the diverging magnetic field lines.

the magnetic field in the upward direction for supersonic $\mathrm{H}^{+}$outflow.

Figure 12 shows schematically how the $\mathrm{H}^{+}$velocity distributions varies with altitude for a collisionless supersonic $\mathrm{H}^{+}$outflow [cf. Holzer et al., 1971]. Starting with a drifting Maxwellian distribution at the exobase $(\sim 4500$ $\mathrm{km})$, the kinetic evolution of the $\mathrm{H}^{+}$velocity distribution produces an anisotropic distribution at higher altitudes with an asymmetry along the field-aligned direction due to the drift. These non-Maxwellian characteristics grow with altitude, and at altitudes greater than about $10 R_{E}$, $T_{\|} / T_{\perp}>20$. Also, above this altitude the asymmetry with respect to the field-aligned direction is sufficient to cause a displacement between the drift velocity and the peak of the velocity distribution. Such large deviations from a Maxwellian have important implications for the stability of the polar wind. In this regard, it should be noted that only a small field-aligned asymmetry is expected for subsonic outflow.

The characteristics of the polar wind described above were determined from steady state models of ionospheric outflow. Recently, however, Singh and Schunk [1982] studied the expansion of a collisionless, electrically neutral, multi-ion plasma into a vacuum because of its possible relevance to the initial expansion of the polar wind. The study indicated that energetic ions are created when a plasma expands. For $\mathrm{H}^{+}$the typical energy gained during the expansion was about $10 k T_{e}$. Since $T_{e}$ probably varies from about $1000^{\circ}$ to $10,000^{\circ} \mathrm{K}$ in the highlatitude topside ionosphere, the energy of the $\mathrm{H}^{+}$ions accelerated in an expanding polar wind should lie in the range of from 1 to $10 \mathrm{eV}$.

The DE-1 measurements made at the higher $L$ values are the most relevant to polar wind, as this is where the upward flowing ion beams were observed. In this region, the ion composition, densities, drift velocities, and fluxes that were deduced from the measurements are in good general agreement with the model predictions. However, because of the uncertainty in the spacecraft potential, it was not possible to determine whether the flow was subsonic or supersonic, i.e., whether it was a polar breeze or a polar wind. This particular conclusion agrees with the earlier DE-1 observations of Gurgiolo and Burch [1982]. Also, because of the wide aperture of the instrument, it was not possible to obtain information on the anisotropic or asymmetric character of the $\mathrm{H}^{+}$velocity distribution.

\subsection{Plasmaspheric Refilling}

Banks et al. [1971] postulated that after flux tube depletion the resulting supersonic field-aligned plasma flows that emerge from the conjugate ionospheres interact at the equator, forming a pair of collisionless electrostatic shocks. Behind the shocks is a relatively high- 
density, high-temperature plasma. As time passes, the volume between the two shocks fills with plasma and the locations of the shocks move down the flux tube toward the ionosphere at a relatively low speed. Eventually, the shock fronts reach their respective critical points and the flow becomes subsonic everywhere along the flux tube. It is at this time that the low speed, collision-dominated transport formulations become valid. Banks et al. [1971] estimated that for the $L=5$ flux tube, the transition from supersonic to subsonic flow would occur about 22 hours after the filling process began.

Schulz and Koons [1972] studied the stability of counterstreaming ion flows by using a one-dimensional analysis based on the ion-acoustic mode. They concluded that collisionless shocks were not likely to form but that ion trapping could occur via wave-particle interactions. Specifically, they predicted that the counterstreaming ion flows were unstable, and therefore thermalization and trapping should occur via pitch angle scattering in waveparticle collisions. With this process, flow energy is converted into thermal energy, and hence the trapped ions constitute a relatively hot background plasma that ultimately attains sufficient density to quench the original two-stream instability. After this time, Coulomb collisions dominate the thermalization process.

More recently, Singh and Schunk [1983] conducted onedimensional numerical simulations that are relevant to the initial refilling of depleted plasmaspheric flux tubes. These simulations support the conclusion by Schulz and Koons [1972] that electrostatic shocks are not likely to form. However, the simulations also indicate that the counterstreaming plasmas are stable with respect to the ion-acoustic mode.

The picture that has emerged from the Singh and Schunk [1983] study is the following. After a magnetic storm, density-shock fronts propagate up from the conjugate ionospheres along depleted flux tubes. However, the shocks are preceded by low-density beams of energetic forerunner ions, which are the first to interact at the equator. A linear instability analysis indicates that the main density shock fronts are stable when they collide and hence will penetrate each other, which can explain the observed TFA counterstreaming flows. However, the counterstreaming energetic forerunners can excite ion cyclotron waves, which in turn can thermalize and trap the energetic forerunners via pitch angle scattering. This process will change the pitch angle distribution of these ions from being field-aligned to perpendicular, with the energy of this perpendicular component being greater than $2.5 \mathrm{eV}$. The low density, warm anisotropic plasma (WAP) measured by DE-1 in the outer plasmasphere can be explained by the above process. It is doubtful that the energetic forerunners would be measured by $D E-1$ when they are in their field-aligned phase, because this phase of the plasma expansion is brief.

Another consequence of the merging of the main density shock fronts is that the merged plasma has a net zero average drift, and therefore the effective temperature along the magnetic field will appear to be very high. However, the temperature perpendicular to the magnetic field will still appear to be low, i.e., it will be a typical ionospheric temperature. Therefore this process cannot explain the DE-1 observations of a high density, thermal, isotropic plasma (TP) in the outer plasmasphere.

\subsection{Instrument Requirements}

The RIMS instrument has shown that instrument bias techniques may be used successfully to overcome spacecraft potential shielding effects and that thermal ions are present in the dayside magnetosphere in nonnegligible concentrations. These ions have a highly anisotropic distribution. However, before critical comparisons can be made with existing polar wind and interhemispheric theories a new generation of instruments with significantly improved energy and angular resolution must be developed. The absence of thermal electron observations severely limits critical comparison of observations with plasma instability and wave interaction models. Indeed, thermal electron instruments with improved resolution comparable to the new generation of thermal ion instruments would be required. On the basis of the comparison between the present RIMS data set and polar wind-interhemispheric models, a qualitative "shopping" list for the new generation magnetospheric thermal plasma instruments has been drawn up and is presented in the appendix.

\section{APPENDIX}

The following section outlines the technical requirements for an instrument to measure the thermal ion and electron distribution functions associated with polar wind, interhemispheric flow and plasmasphere refilling. Although it may be impractical for a single instrument to satisfy all these requirements, an attempt should be made on future missions into the mid- and high-latitude middle magnetosphere to provide a complementary set of instruments with the following characteristics.

\section{Ion Instrument}

1. Must measure the distribution function of the principal ion species, in the range $0.02-100 \mathrm{eV}, \mathrm{H}^{+}$, and $\mathrm{He}^{+}$, and $\mathrm{O}^{+}$. Measurements of minor ions $\left(\mathrm{He}^{++}, \mathrm{O}^{++}, \mathrm{N}^{+}\right)$ would be desirable but is not a high priority.

2. In order to achieve item 1 the instrument must either be mounted on a spacecraft where active control of spacecraft potential is implemented or must have the capability to apply a bias voltage to overcome the positive spacecraft potential experienced beyond the plasmasphere.

3. Angular resolution (real pitch angle resolution) should be less than $2^{\circ}$, in order that thermal source cone structures can be studied in the magnetosphere.

4. Energy resolution should not be worse than $5 \%$ over the entire energy scan.

5. Differential energy analysis would enable 4 to be readily achieved, and electrostatic lens systems could well be used to achieve 3 and yet give reasonable fluxes to ensure that reasonable time resolution is maintained. 


\section{Electron Instrument}

1. The energy and angular resolution specified for the ions also applies to the electrons.

2. The trade-offs made in using the same physical analyzer for measuring ions and electrons is too great, i.e., the difference in relative sensitivity between electrons and ions ranging from 10 to 100 and the completely opposite bias voltage requirements to counter a positive spacecraft potential. Hence the electron instrument should be a separate instrument mounted as far as possible from the ion instruments sheath perturbations.

Acknowledgements. We thank S. Shawhan for helpful discussions. We acknowledge the computer support provided by the Space Plasma Computer Analysis Network at NASA's Marshall Space Flight Center. This research was supported by NASA grant NAGW-77 and NSF grant ATM-8217138 to Utah State University.

The Editor thanks J. Burch and S. Sanatani for their assistance in evaluating this paper.

\section{REFERENCES}

Banks, P. M., and T. E. Holzer, High-latitude plasma transport: The polar wind, J. Geophys. Res., 74, 6317, 1969.

Banks, P. M., A. F. Nagy, and W. I. Axford, Dynamical behavior of thermal protons in the mid-latitude ionosphere and magnetosphere, Planet. Space Sci., 19, 1053-1067, 1971.

Bezrukikh, V. V., and K. I. Gringauz, The hot zone in the outer plasmasphere of the earth, J. Atmos. Terr. Phys., 38, 1085-1091, 1976.

Carpenter, D. L., and C. G. Park, On what ionospheric workers should know about the plasmapause-plasmasphere, Rev. Geophys. Space Phys., 11, 133, 1973.

Chappell, C. R., Recent satellite measurements of the morphology and dynamics of the plasmasphere, Rev. Geophys. Space Phys., 10, 51, 1972.

Chappell, C. R., Initial observations of thermal plasma composition and energetics from Dynamics Explorer-1, Geophys. Res. Lett., 9, 929-932, 1982.

Chappell, C. R., S. A. Fields, C. R. Baugher, J. H. Hoffman, W. B. Hanson, W. W. Wright, and H. D. Hammack, The retarding ion mass spectrometer on Dynamics Explorer-A, Space Sci., Instrum., 5, 477, 1981.

Chappell, C. R., J. L. Green, J. F. E. Johnson, and J. H. Waite, Jr., Pitch angle variations in magnetospheric thermal plasmaInitial observations from Dynamics Explorer 1, Geophys. Res. Lett., 9, 933-936, 1982.

Comfort, R. H., C. R. Baugher, and C. R. Chappell, Use of the thin sheath approximation for obtaining ion temperatures from ISEE 1 limited aperture RPA, J. Geophys. Res., 87, 5109, $1982 a$.

Comfort, R. H., J. L. Horwitz, C. R. Chappell, J. H. Waite, J. F. E. Johnson, S. D. Shawhan, and R. L. Huff, DE 1 Plasma densities from particle and wave measurements, EoS Trans. AGU; 63, 1056, $1982 b$.

Decreau, P. M. E., C. Beghin, and M. Parrot, Global characterizations of the cold plasma in the equatorial plasmapause region as deduced from the GEOS 1 mutual impedance probe, J. Geophys. Res., 87, 695, 1982.

Foster, J. C., C. C. Park, L. H. Brace, J. R. Burrows, J. H. Hoffman, E. J. Maier, and J. H. Whitaker, Plasmapause signatures in the ionosphere and magnetosphere, J. Geophys. Res., 83, $1175,1978$.

Gurgiolo, C. and J. L. Burch, DE 1 observations of the polar wind-a heated and an unheated component, Geophys. Res. Lett., 9, 945-948, 1982.

Hoffman, J. H., and W. H. Dodson, Light ion concentrations and fluxes in the polar regions during magnetically quiet times,
J. Geophys. Res., 85, 626, 1980.

Holzer, T. E., J. A. Fedder, and P. M. Banks, A comparison of kinetic and hydromagnetic models of an expanding ionexosphere, J. Geophys. Res., 76, 2453, 1971.

Horwitz, J. L., and C. R. Chappell, Observations of warm plasma in the dayside plasma trough at geosynchronous orbit, J. Geophys. Res., 84, 7075-7090, 1979.

Horwitz, J. L., C. R. Baugher, C. R. Chappell, E. G. Shelley, and D. T. Young, Pancake pitch angle distributions in warm ions observed with ISEE 1,J. Geophys. Res., 86, 3311, $1981 a$.

Horwitz, J. L., C. R. Baugher, C. R. Chappell, E. G. Shelley, D. T. Young, and R. R. Anderson, ISEE 1 Observations of thermal plasma in the vicinity of the plasmasphere during periods of quieting magnetic activity, J. Geophys. Res., 86, 9989-10,001, $1981 b$.

Horwitz, J. L., C. R. Baugher, C. R. Chappell, E. G. Shelley, and D. T. Young, Conical pitch angle distributions of very lowenergy ion fluxes observed by ISEE 1, J. Geophys. Res., 87, 2311-2320, 1982.

Lemaire, J., and M. Scherer, Exospheric models of the topside ionosphere, Space Sci. Rev., 15, 591, 1974.

Marubashi, K., Escape of the polar-ionospheric plasma into the magnetospheric tail, Rep. Ionos. Space Res. Jpn., 24, 322, 1970.

Maynard, N. C., T. L. Aggson, and J. P. Heppner, Plasmaspheric electric field as measured by ISEE 1, EoS Trans. AGU, 64, 103, 1983.

Park, C. G., Some features of plasma distribution in the plasmasphere deduced from the antarctic whistlers, $J$. Geophys. Res., 79, 169-173, 1974.

Raitt, W. J., and R. W. Schunk, Composition and characteristics of the polar wind, in Energetic Ion Composition in the Earth's Magnetosphere, edited by R. Johnson, Terra Scientific Publishing Company, Tokyo, in press, 1983.

Raitt, W. J., R. W. Schunk, and P. M. Banks, A comparison of the temperature and density structure in high and low speed thermal proton flows, Planet. Space Sci., 23, 1103, 1975.

Raitt, W. J., R. W. Schunk, and P. M. Banks, The influence of convection electric fields on thermal proton outflow from the ionosphere, Planet. Space Sci., 25, 291, 1977.

Rycroft, M. J. and J. Lemaire, Physics of the plasmapause, Ann. Geophys., 31, 1, 1975.

Schulz, M., and H. C. Koons, Thermalization of colliding ion streams beyond the plasmapause, J. Geophys. Res., 77, 248-254, 1972.

Schunk, R. W., and D. S. Watkins, Proton temperature anisotropy in the polar wind, J. Geophys. Res., 87, 171, 1982.

Singh, N., and C. R. Baugher, Sheath effects on current collection by particle detectors with narrow acceptance angles, Space Sci., Instrum., 5, 295, 1981.

Singh, N., and R. W. Schunk, Numerical calculations relevant to the initial expansion of the polar wind, J. Geophys. Res., 87, 9154-9170, 1982.

Singh, N., and R. W. Schunk, Numerical simulations of counterstreaming plasmas and their relevance to interhemispheric flows, J. Geophys. Res., 88, in press, 1983.

Sojka, J. J., and R. W. Schunk, Analysis and interpretation techniques for the RIMS experiment on DE-A, Report, Center for Atmospheric and Space Sciences, Utah State University, Logan, 1982.

Whipple, E. C., J. M. Warnock, and R. H. Winkler, Effect of satellite potential on direct ion density measurements through the plasmapause, J. Geophys. Res., 79, 179-186, 1974.

C. R. Chappell, J. F. E. Johnson, and J. H. Waite, Magnetospheric Physics Branch, Space Science Laboratory, Marshall Space Flight Center, Huntsville, AL 35812.

R. W. Schunk and J. J. Sojka, Center for Atmospheric and Space Sciences, Utah State University, UMC 34, Logan, UT 84322.

(Received April 4, 1983; revised July 5,1983 ; accepted July 6,1983 .) 